\title{
A STRONGER DERIVED TORELLI THEOREM FOR K3 SURFACES
}

\author{
MAX LIEBLICH AND MARTIN OLSSON
}

\begin{abstract}
In an earlier paper the notion of a filtered derived equivalence was introduced, and it was shown that if two K3 surfaces admit such an equivalence then they are isomorphic. In this paper we study more refined aspects of filtered derived equivalences related to the action on the cohomological realizations of the Mukai motive. It is shown that if a filtered derived equivalence between K3 surfaces also preserves ample cones then one can find an isomorphism that induces the same map as the equivalence on the cohomological realizations.
\end{abstract}

\section{Contents}

1. Introduction

2. Strongly filtered equivalences

3. Moduli spaces of K3 surfaces

4. Deformations of autoequivalences

5. A remark on reduction types

6. Supersingular reduction

7. Specialization

8. Proof of Theorem 1.2

9. Characteristic 0

10. Bypassing Hodge theory

\section{InTRODUCTION}

1.1. Let $k$ be an algebraically closed field of odd characteristic and let $X$ and $Y$ be K3 surfaces over $k$. Let

$$
\Phi: D(X) \rightarrow D(Y)
$$

be an equivalence between their bounded triangulated categories of coherent sheaves given by a Fourier-Mukai kernel $P \in D(X \times Y)$, so $\Phi$ is the functor given by sending $M \in D(X)$ to

$$
R \operatorname{pr}_{2 *}\left(L \operatorname{pr}_{1}^{*} M \otimes^{\mathbb{L}} P\right) .
$$


As discussed in [15, 2.9] the kernel $P$ also induces an isomorphism on rational Chow groups modulo numerical equivalence

$$
\Phi_{P}^{A^{*}}: A^{*}(X)_{\text {num }, \mathbb{Q}} \rightarrow A^{*}(Y)_{\text {num }, \mathbb{Q}} .
$$

We can consider how a given equivalence $\Phi$ interacts with the codimension filtration on $A^{*}$, or how it acts on the ample cone of $X$ inside $A^{1}(X)$. The underlying philosophy of this work is that tracking filtrations and ample cones (in ways we will make precise in Section 2) gives a semi-linear algebraic gadget that behaves a lot like a Hodge structure. In Section 2 we will define a notion of strongly filtered for an equivalence $\Phi$ that imposes conditions reminiscent of the classical Torelli theorem for K3 surfaces.

With this in mind, the purpose of this paper is to prove the following result.

Theorem 1.2. If $\Phi_{P}: D(X) \rightarrow D(Y)$ is a strongly filtered equivalence then there exists an isomorphism $\sigma: X \rightarrow Y$ such that the maps on the crystalline and étale realizations of the Mukai motive induced by $\Phi_{P}$ and $\sigma$ agree.

For the definition of the realizations of the Mukai motive see [15, §2]. In [15, Proof of 6.2 ] it is shown that any filtered equivalence can be modified to be strongly filtered. As a consequence, we get a new proof of the following result.

Theorem $1.3([15,6.1])$. If $\Phi_{P}^{A^{*}}$ preserves the codimension filtrations on $A^{*}(X)_{\text {num, } \mathbb{Q}}$ and $A^{*}(Y)_{\text {num, } \mathbb{Q}}$ then $X$ and $Y$ are isomorphic.

Whereas the original proof of Theorem 1.3 relied heavily on liftings to characteristic 0 and Hodge theory, the proof presented here works primarily in positive characteristic using algebraic methods.

In Section 8 we present a proof of Theorem 1.2 using certain results about "Kulikov models" in positive characteristic (see Section 5). This argument implicitly uses Hodge theory which is an ingredient in the proof of Theorem 5.3. In Section 9 we discuss a characteristic 0 variant of Theorem 1.2, and finally the last section 10 we explain how to bypass the use of the Hodge theory ingredient of Theorem 5.3. This makes the argument entirely algebraic, except for the Hodge theory aspects of the proof of the Tate conjecture. This also gives a different algebraic perspective on the statement that any Fourier-Mukai partner of a K3 surface is a moduli space of sheaves, essentially inverting the methods of [15].

The bulk of this paper is devoted to proving Theorem 1.2. The basic idea is to consider a certain moduli stack $\mathscr{S}_{d}$ classifying data $((X, \lambda), Y, P)$ consisting of a primitively polarized K3 surface $(X, \lambda)$ with polarization of some degree $d$, a second K3 surface $Y$, and a complex $P \in D(X \times Y)$ defining a strongly filtered Fourier-Mukai equivalence $\Phi_{P}: D(X) \rightarrow D(Y)$. The precise definition is given in Section [3, where it is shown that $\mathscr{S}_{d}$ is an algebraic stack which is naturally a $\mathbb{G}_{m}$-gerbe over a Deligne-Mumford stack $\overline{\mathscr{S}}_{d}$ étale over the stack $\mathscr{M}_{d}$ classifying primitively polarized K3 surfaces of degree $d$. The map $\overline{\mathscr{S}}_{d} \rightarrow \mathscr{M}_{d}$ is induced by the map sending a collection $((X, \lambda), Y, P)$ to $(X, \lambda)$. We then study the locus of points in $\mathscr{S}_{d}$ where Theorem 1.2 holds showing that it is stable under both generization and specialization. From this it follows that it suffices to consider the case when $X$ and $Y$ are supersingular where we can use Ogus' crystalline Torelli theorem [24, Theorem I]. 
Remark 1.4. Our restriction to odd characteristic is because we appeal to the Tate conjecture for K3 surfaces, proven in odd characteristics by Charles, Maulik, and Pera [7, 19, 22], which at present is not known in characteristic 2 .

1.5. (Acknowledgements) Lieblich partially supported by NSF CAREER Grant DMS-1056129 and Olsson partially supported by NSF grant DMS-1303173 and a grant from The Simons Foundation. Olsson is grateful to F. Charles for inspiring conversations at the Simons Symposium "Geometry Over Nonclosed Fields" which led to results of this paper. We also thank E. Macrì D. Maulik, and K. Pera for useful correspondence.

\section{Strongly FILTEREd EQUivalenCES}

2.1. Let $X$ and $Y$ be $K 3$ surfaces over an algebraically closed field $k$ and let $P \in D(X \times Y)$ be an object defining an equivalence

$$
\Phi_{P}: D(X) \rightarrow D(Y)
$$

and let

$$
\Phi_{P}^{A_{\text {num }, \mathbb{Q}}^{*}}: A^{*}(X)_{\text {num }, \mathbb{Q}} \rightarrow A^{*}(Y)_{\text {num }, \mathbb{Q}}
$$

denote the induced map on Chow groups modulo numerical equivalence and tensored with $\mathbb{Q}$. We say that $\Phi_{P}$ is filtered (resp. strongly filtered, resp. Torelli) if $\Phi_{P}^{A_{\text {num,Q }}^{*}}$ preserves the codimension filtration (resp. is filtered, sends $(1,0,0)$ to $(1,0,0)$, and sends the ample cone of $X$ to plus or minus the ample cone of $Y$; resp. is filtered, sends $(1,0,0)$ to $\pm(1,0,0)$, and sends the ample cone of $X$ to the ample cone of $Y$ ).

Remark 2.2. Note that if $P$ is strongly filtered then either $P$ or $P[1]$ is Torelli. If $P$ is Torelli then either $P$ or $P[1]$ is strongly filtered.

Remark 2.3. Note that $A^{1}(X)$ is the orthogonal complement of $A^{0}(X) \oplus A^{2}(X)$ and similarly for $Y$. This implies that if $\Phi_{P}$ is filtered and sends $(1,0,0)$ to $\pm(1,0,0)$ then $\Phi_{P}\left(A^{1}(X)_{\text {num, } \mathbb{Q}}\right) \subset$ $A^{1}(Y)_{\text {num, }} \mathbb{Q}$.

Remark 2.4. It is shown in [15, 6.2] that if $\Phi_{P}: D(X) \rightarrow D(Y)$ is a filtered equivalence, then there exists a strongly filtered equivalence $\Phi: D(X) \rightarrow D(Y)$. In fact it is shown there that $\Phi$ can be obtained from $\Phi_{P}$ by composing with a sequence of shifts, twists by line bundles, and spherical twists along (-2)-curves.

2.5. As noted in [15, 2.11] an equivalence $\Phi_{P}$ is filtered if and only if the induced map on Chow groups

$$
\Phi_{P}^{A_{\text {num }, \mathbb{Q}}^{*}}: A^{*}(X)_{\text {num }, \mathbb{Q}} \rightarrow A^{*}(Y)_{\text {num }, \mathbb{Q}}
$$

sends $A^{2}(X)_{\text {num }, \mathbb{Q}}$ to $A^{2}(X)_{\text {num, }, \mathbb{Q}}$.

Lemma 2.6. Let $\ell$ be a prime invertible in $k$, let $\widetilde{H}\left(X, \mathbb{Q}_{\ell}\right)$ (resp. $\widetilde{H}\left(Y, \mathbb{Q}_{\ell}\right)$ ) denote the $\mathbb{Q}_{\ell}$-realization of the Mukai motive of $X$ (resp. $Y$ ) as defined in [15, 2.4], and let

$$
\Phi_{P}^{\text {ét }}: \widetilde{H}\left(X, \mathbb{Q}_{\ell}\right) \rightarrow \widetilde{H}\left(Y, \mathbb{Q}_{\ell}\right)
$$

denote the isomorphism defined by $P$. Then $\Phi_{P}$ is filtered if and only if $\Phi_{P}^{\mathrm{et}}$ preserves the filtrations by degree on $\widetilde{H}\left(X, \mathbb{Q}_{\ell}\right)$ and $\widetilde{H}\left(Y, \mathbb{Q}_{\ell}\right)$. 
Proof. By the same reasoning as in $[15,2.4]$ the map $\Phi_{P}^{\text {ét }}$ is filtered if and only if

$$
\Phi_{P}^{\text {ét }}\left(H^{4}\left(X, \mathbb{Q}_{\ell}\right)\right)=H^{4}\left(Y, \mathbb{Q}_{\ell}\right) .
$$

Since the cycle class maps

$$
A^{2}(X)_{\text {num, }, \mathbb{Q}} \otimes_{\mathbb{Q}} \mathbb{Q}_{\ell} \rightarrow H^{4}\left(X, \mathbb{Q}_{\ell}\right), \quad A^{2}(Y)_{\text {num, }, \mathbb{Q}} \otimes_{\mathbb{Q}} \mathbb{Q}_{\ell} \rightarrow H^{4}\left(Y, \mathbb{Q}_{\ell}\right)
$$

are isomorphisms and the maps $\Phi_{P}$ and $\Phi_{P}^{\text {ét }}$ are compatible in the sense of [15, 2.10] it follows that if $\Phi_{P}$ is filtered then so is $\Phi_{P}^{\text {ét }}$. Conversely if $\Phi_{P}^{\text {ét }}$ is filtered then since the cycle class maps

$$
A^{*}(X)_{\text {num }, \mathbb{Q}} \rightarrow \widetilde{H}\left(X, \mathbb{Q}_{\ell}\right), \quad A^{*}(Y)_{\text {num }, \mathbb{Q}} \rightarrow \widetilde{H}\left(Y, \mathbb{Q}_{\ell}\right)
$$

are injective it follows that $\Phi_{P}$ is also filtered.

Remark 2.7. The same proof as in Lemma 2.6 gives variant results for crystalline cohomology and in characteristic 0 de Rham cohomology.

The condition that $\Phi_{P}$ takes the ample cone to plus or minus the ample cone appears more subtle. A useful observation in this regard is the following.

Lemma 2.8. Let $P \in D(X \times Y)$ be an object defining a filtered equivalence $\Phi_{P}: D(X) \rightarrow$ $D(Y)$ such that $\Phi_{P}^{A_{\mathrm{num}}^{*}}$ sends $(1,0,0)$ to $(1,0,0)$. Then $\Phi_{P}$ is strongly filtered if and only if for some ample invertible sheaf $L$ on $X$ the class $\Phi_{P}^{A_{\text {num }}^{*}}(L) \in N S(Y)_{\mathbb{Q}}$ is plus or minus an ample class.

Proof. Following [24, p. 366] define

$$
V_{X}:=\left\{x \in N S(X)_{\mathbb{R}} \mid x^{2}>0, \text { and }\langle x, \delta\rangle \neq 0 \text { for all } \delta \in N S(X) \text { with } \delta^{2}=-2\right\},
$$

and define $V_{Y}$ similarly. Since $\Phi_{P}^{A_{\text {num }}^{*}}$ is an isometry it induces an isomorphism

$$
\sigma: V_{X} \rightarrow V_{Y}
$$

By [24, Proposition 1.10 and Remark 1.10.9] the ample cone $C_{X}$ (resp. $C_{Y}$ ) of $X$ (resp. $Y$ ) is a connected component of $V_{X}$ (resp. $\left.V_{Y}\right)$ and therefore either $\sigma\left(C_{X}\right) \cap C_{Y}=\emptyset$ or $\sigma\left(C_{X}\right)=C_{Y}$, and similarly $\sigma\left(-C_{X}\right) \cap C_{Y}=\emptyset$ or $\sigma\left(-C_{X}\right)=C_{Y}$.

Proposition 2.9. Let $X$ and $Y$ be K3-surfaces over a scheme $S$ and let $P \in D\left(X \times_{S} Y\right)$ be a relatively perfect complex. Assume that $X / S$ is projective. Then the set of points $s \in S$ for which the induced transformation on the derived category of the geometric fibers

$$
\Phi_{P_{\bar{s}}}: D\left(X_{\bar{s}}\right) \rightarrow D\left(Y_{\bar{s}}\right)
$$

is a strongly filtered equivalence is an open subset of $S$.

Proof. By a standard reduction we may assume that $S$ is of finite type over $\mathbb{Z}$.

First note that the condition that $\Phi_{P_{\bar{s}}}$ is an equivalence is an open condition. Indeed as described in [15, discussion preceding 3.3] there exists a morphism of $S$-perfect complexes $\epsilon: P_{1} \rightarrow P_{2}$ in $D\left(X \times_{S} Y\right)$ such that $\Phi_{P_{\bar{s}}}$ is an equivalence if and only if $\epsilon_{\bar{s}}: P_{1, \bar{s}} \rightarrow P_{2, \bar{s}}$ is an isomorphism in $D\left(X_{\bar{s}} \times Y_{\bar{s}}\right)$ (in loc. cit. we considered two maps of perfect complexes but one can just take the direct sum of these to get $\epsilon$ ). Let $Q$ be the cone of $\epsilon$, and let $Z \subset X \times{ }_{S} Y$ be the support of the cohomology sheaves of $Q$. Then the image of $Q$ in $S$ is closed and the 
complement of this image is the maximal open set over which the fiber transformations $\Phi_{P_{\bar{s}}}$ are equivalences.

Replacing $S$ by an open set we may therefore assume that $\Phi_{P_{\bar{s}}}$ is an equivalence in every fiber.

Next we show that the condition that $\Phi_{P}$ is filtered is an open and closed condition. For this we may assume we have a prime $\ell$ invertible in $S$. Let $f_{X}: X \rightarrow S$ (resp. $f_{Y}: Y \rightarrow S$ ) be the structure morphism. Define $\widetilde{\mathscr{H}}_{X / S}$ to be the lisse $\mathbb{Q}_{\ell}$-sheaf on $S$ given by

$$
\widetilde{\mathscr{H}}_{X / S}:=\left(R^{0} f_{X *} \mathbb{Q}_{\ell}(-1)\right) \oplus\left(R^{2} f_{X *} \mathbb{Q}_{\ell}\right) \oplus\left(R^{4} f_{X *} \mathbb{Q}_{\ell}\right)(1)
$$

and define $\widetilde{\mathscr{H}}_{Y / S}$ similarly. The kernel $P$ then induces a morphism of lisse sheaves

$$
\Phi_{P / S}^{\text {ét,l }}: \widetilde{\mathscr{H}}_{X / S} \rightarrow \widetilde{\mathscr{H}}_{Y / S}
$$

whose restriction to each geometric fiber is the map on the $\mathbb{Q}_{\ell}$-realization of the Mukai motive as in [15, 2.4]. In particular, $\Phi_{P / S}^{\text {ét, } \ell}$ is an isomorphism. By Lemma 2.6 for every geometric point $\bar{s} \rightarrow S$ the map $\Phi_{P_{\bar{s}}}$ is filtered if and only if the stalk $\Phi_{P / S, \bar{s}}^{\text {ét, } \ell}$ preserves the filtrations on $\widetilde{\mathscr{H}}_{X / S}$ and $\widetilde{\mathscr{H}}_{Y / S}$. In particular this is an open and closed condition on $S$. Shrinking on $S$ if necessary we may therefore further assume that $\Phi_{P_{\bar{s}}}$ is filtered for every geometric point $\bar{s} \rightarrow S$.

It remains to show that in this case the set of points $s$ for which $\Phi_{P}$ takes the ample cone $C_{X_{\bar{s}}}$ of $X_{\bar{s}}$ to $\pm C_{Y_{\bar{s}}}$ is an open subset of $S$. For this we can choose, by our assumption that $X / S$ is projective, a relatively ample invertible sheaf $L$ on $X$. Define

$$
M:=\operatorname{det}\left(\operatorname{Rpr}_{2 *}\left(\operatorname{Lpr}_{1}^{*}(L) \otimes P\right)\right),
$$

an invertible sheaf on $Y$. Then by Lemma 2.8 for a point $s \in S$ the transformation $\Phi_{P_{\bar{s}}}$ is strongly filtered if and only if the restriction of $M$ to the fiber $Y_{\bar{s}}$ is plus or minus the class of an ample divisor. By openness of the ample locus [9, III, 4.7.1] we get that being strongly filtered is an open condition.

Proposition 2.10. Let $P \in D(X \times Y)$ be a complex such that the induced transformation

$$
\Phi_{P}^{A_{\text {num }, \mathbb{Q}}^{*}}: A^{*}(X)_{\text {num }, \mathbb{Q}} \rightarrow A^{*}(X)_{\text {num }, \mathbb{Q}}
$$

preserves the codimension filtration, takes $(1,0,0)$ to $(1,0,0)$, and takes the ample cone of $X$ to plus or minus the ample cone of $Y$ (so $P$ does not necessarily define an equivalence but otherwise behaves like a strongly filtered Fourier-Mukai equivalence). Suppose there exists an equivalence $\Phi_{Q}: D(X) \rightarrow D(Y)$ which is Torelli, and such that the induced map NS(X) $\rightarrow$ $N S(Y)$ agrees with the map defined by $\pm \Phi_{P}$. Then $\Phi_{P}^{A_{\text {num, }}^{*}}$ preserves the ample cones.

Proof. Suppose that $\Phi_{P}$ takes the ample cone of $X$ to the negative of the ample cone of $Y$. Consider the auto-equivalence $\Phi:=\Phi_{Q}^{-1} \circ \Phi_{P[1]}$ of $D(X)$. The induced automorphism

$$
\Phi^{A_{\text {num }, \mathbb{Q}}^{*}}: A^{*}(X)_{\text {num }, \mathbb{Q}} \rightarrow A^{*}(X)_{\text {num, } \mathbb{Q}}
$$

then preserves the codimension filtration, Mukai pairing, and is the identity on $N S(X)_{\text {num, } \mathbb{Q}}$ and multiplication by -1 on $A^{0}(X)_{\text {num, } \mathbb{Q}}$ and $A^{2}(X)_{\text {num, }}$. By the compatibility of $\Phi$ with 
the Mukai pairing this implies that for any $H \in N S(X)$ we have

$$
-H^{2}=\Phi\langle(0, H, 0),(0, H, 0)\rangle=\langle(0, H, 0),(0, H, 0)\rangle=H^{2},
$$

which is a contradiction. Thus $\Phi_{P[1]}$ must take $(0,0,1)$ to $(0,0,1)$ which implies that $\Phi_{P[1]}$ takes $(1,0,0)$ to $(1,0,0)$, a contradiction.

\section{Moduli spaces of K3 surfaces}

3.1. For an integer $d$ invertible in $k$ let $\mathscr{M}_{d}$ denote the stack over $k$ whose fiber over a scheme $T$ is the groupoid of pairs $(X, \lambda)$ where $X / T$ is a proper smooth algebraic space all of whose geometric fibers are K3 surfaces and $\lambda: T \rightarrow \mathrm{Pic}_{X / T}$ is a morphism to the relative Picard functor such that in every geometric fiber $\lambda$ is given by a primitive ample line bundle $L_{\lambda}$ whose self-intersection is $2 d$. The following theorem summarizes the properties of the stack $\mathscr{M}_{d}$ that we will need.

Theorem 3.2. (i) $\mathscr{M}_{d}$ is a Deligne-Mumford stack, smooth over $k$ of relative dimension 19.

(ii) If $p \geq 3$ and $p^{2} \nmid d$ then the geometric fiber of $\mathscr{M}_{d}$ is irreducible.

(iii) The locus $\mathscr{M}_{d, \infty} \subset \mathscr{M}_{d}$ classifying supersingular $K 3$ surfaces is closed of dimension $\geq 9$.

Proof. A review of (i) and (iii) can be found in [23, p. 1]. Statement (ii) can be found in [16, $2.10(3)]$.

Remark 3.3. The stack $\mathscr{M}_{d}$ is defined over $\mathbb{Z}$, and it follows from (ii) that the geometric generic fiber of $\mathscr{M}_{d}$ is irreducible (this follows also from the Torelli theorem over $\mathbb{C}$ and the resulting description of $\mathscr{M}_{d, \mathbb{C}}$ as a period space). Furthermore over $\mathbb{Z}[1 / d]$ the stack $\mathscr{M}_{d}$ is smooth. In what follows we denote this stack over $\mathbb{Z}[1 / d]$ by $\mathscr{M}_{d, \mathbb{Z}[1 / 2]}$ and reserve the notation $\mathscr{M}_{d}$ for its reduction to $k$.

Remark 3.4. Note that in the definition of $\mathscr{M}_{d}$ we consider ample invertible sheaves, and don't allow contractions in the corresponding morphism to projective space.

3.5. Let $\mathscr{S}_{d}$ denote the fibered category over $k$ whose fiber over a scheme $S$ is the groupoid of collections of data

$$
((X, \lambda), Y, P)
$$

where $(X, \lambda) \in \mathscr{M}_{d}(S)$ is a polarized K3 surface, $Y / S$ is a second K3 surface over $S$, and $P \in D\left(X \times_{S} Y\right)$ is an $S$-perfect complex such that for every geometric point $\bar{s} \rightarrow S$ the induced functor

$$
\Phi^{P_{\bar{s}}}: D\left(X_{\bar{s}}\right) \rightarrow D\left(Y_{\bar{s}}\right)
$$

is strongly filtered.

Theorem 3.6. The fibered category $\mathscr{S}_{d}$ is an algebraic stack locally of finite type over $k$.

Proof. By fppf descent for algebraic spaces we have descent for both polarized and unpolarized K3 surfaces.

To verify descent for the kernels $P$, consider an object 3.5.1 over a scheme $S$. Let $P^{\vee}$ denote $\mathscr{R} \operatorname{Hom}\left(P, \mathscr{O}_{X}\right)$. Since $P$ is a perfect complex we have $\mathscr{R} H o m(P, P) \simeq P^{\vee} \otimes P$. By $[14$, 
2.1.10] it suffices to show that for all geometric points $\bar{s} \rightarrow S$ we have $H^{i}\left(X_{\bar{s}} \times Y_{\bar{s}}, P_{\bar{s}}^{\vee} \otimes P_{\bar{s}}\right)=0$ for $i<0$. This follows from the following result (we discuss Hochschild cohomology further in Section 4 below):

Lemma 3.7 ([26, 5.6], [10, 5.1.8]). Let $X$ and $Y$ be K3 surfaces over an algebraically closed field $k$, and let $P \in D(X \times Y)$ be a complex defining a Fourier-Mukai equivalence $\Phi_{P}$ : $D(X) \rightarrow D(Y)$. Denote by $H H^{*}(X)$ the Hochschild cohomology of $X$ defined as

$$
\operatorname{RHom}_{X \times X}\left(\Delta_{*} \mathscr{O}_{X}, \Delta_{*} \mathscr{O}_{X}\right) .
$$

(i) There is a canonical isomorphism $\operatorname{Ext}_{X \times Y}^{*}(P, P) \simeq H H^{*}(X)$.

(ii) $\operatorname{Ext}_{X \times Y}^{i}(P, P)=0$ for $i<0$ and $i=1$.

(iii) The natural map $k \rightarrow \operatorname{Ext}_{X \times Y}^{0}(P, P)$ is an isomorphism.

Proof. Statement (i) is [26, 5.6]. Statements (ii) and (iii) follow immediately from this, since $H H^{1}(X)=0$ for a $\mathrm{K} 3$ surface.

Next we show that for an object (3.5.1) the polarization $\lambda$ on $X$ induces a polarization $\lambda_{Y}$ on $Y$. To define $\lambda_{Y}$ we may work étale locally on $S$ so may assume there exists an ample invertible sheaf $L$ on $X$ defining $\lambda$. The complex

$$
\Phi_{P}(L):=R \operatorname{pr}_{2 *}\left(\operatorname{pr}_{1}^{*} L \otimes^{\mathbb{L}} P\right)
$$

is $S$-perfect, and therefore a perfect complex on $Y$. Let $M$ denote the determinant of $\Phi_{P}(L)$, so $M$ is an invertible sheaf on $Y$. By our assumption that $\Phi^{P_{s}}$ is strongly filtered for all $s \in S$, the restriction of $M$ to any fiber is either ample or antiample. It follows that either $M$ or $M^{\vee}$ is a relatively ample invertible sheaf and we define $\lambda_{Y}$ to be the resulting polarization on $Y$. Note that this does not depend on the choice of line bundle $L$ representing $\lambda$ and therefore by descent $\lambda_{Y}$ is defined even when no such $L$ exists.

The degree of $\lambda_{Y}$ is equal to $d$. Indeed if $s \in S$ is a point then since $\Phi^{P_{s}}$ is strongly filtered the induced map $N S\left(X_{\bar{s}}\right) \rightarrow N S\left(Y_{\bar{s}}\right)$ is compatible with the intersection pairings and therefore $\lambda_{Y}^{2}=\lambda^{2}=2 d$.

From this we deduce that $\mathscr{S}_{d}$ is algebraic as follows. We have a morphism

$$
\mathscr{S}_{d} \rightarrow \mathscr{M}_{d} \times \mathscr{M}_{d}, \quad((X, \lambda), Y, P) \mapsto\left((X, \lambda),\left(Y, \lambda_{Y}\right)\right),
$$

and $\mathscr{M}_{d} \times \mathscr{M}_{d}$ is an algebraic stack. Let $\mathscr{X}$ (resp. $\mathscr{Y}$ ) denote the pullback to $\mathscr{M}_{d} \times \mathscr{M}_{d}$ of the universal family over the first factor (resp. second factor). Sending a triple $((X, \lambda), Y, P)$ to $P$ then realizes $\mathscr{S}_{d}$ as an open substack of the stack over $\mathscr{M}_{d} \times \mathscr{M}_{d}$ of simple universally gluable complexes on $\mathscr{X} \times \mathscr{M}_{d} \times \mathscr{M}_{d} \mathscr{Y}$ (see for example [15, §5]).

3.8. Observe that for any object $((X, \lambda), Y, P) \in \mathscr{S}_{d}$ over a scheme $S$ there is an inclusion

$$
\mathbb{G}_{m} \hookrightarrow \underline{\text { Aut }}_{\mathscr{P}_{d}}((X, \lambda), Y, P)
$$

giving by scalar multiplication by $P$. We can therefore form the rigidification of $\mathscr{S}_{d}$ with respect to $\mathbb{G}_{m}$ (see for example $[2, \S 5]$ ) to get a morphism

$$
g: \mathscr{S}_{d} \rightarrow \overline{\mathscr{S}}_{d}
$$


realizing $\mathscr{S}_{d}$ as a $\mathbb{G}_{m}$-gerbe over another algebraic stack $\overline{\mathscr{S}}_{d}$. By the universal property of rigidification the map $\mathscr{S}_{d} \rightarrow \mathscr{M}_{d}$ sending $((X, \lambda), Y, P)$ to $(X, \lambda)$ induces a morphism

$$
\pi: \overline{\mathscr{S}}_{d} \rightarrow \mathscr{M}_{d}
$$

Theorem 3.9. The stack $\overline{\mathscr{S}}_{d}$ is Deligne-Mumford and the map (3.8.1) is étale.

Proof. Consider the map [3.7.1. By the universal property of rigidification this induces a morphism

$$
q: \overline{\mathscr{S}}_{d} \rightarrow \mathscr{M}_{d} \times \mathscr{M}_{d}
$$

Since $\mathscr{M}_{d} \times \mathscr{M}_{d}$ is Deligne-Mumford, to prove that $\overline{\mathscr{S}}_{d}$ is a Deligne-Mumford stack it suffices to show that $q$ is representable. This follows from Lemma 3.7 (iii) which implies that for any object $((X, \lambda), Y, P)$ over a scheme $S$ the automorphisms of this object which map under $q$ to the identity are given by scalar multiplication on $P$ by elements of $\mathscr{O}_{S}^{*}$.

It remains to show that the map (3.8.1) is étale, and for this it suffices to show that it is formally étale.

Let $A \rightarrow A_{0}$ be a surjective map of artinian local rings with kernel $I$ annhilated by the maximal ideal of $A$, and let $k$ denote the residue field of $A_{0}$ so $I$ can be viewed as a $k$-vector space. Let $\left(\left(X_{0}, \lambda_{0}\right), Y_{0}, P_{0}\right) \in \mathscr{S}_{d}\left(A_{0}\right)$ be an object and let $(X, \lambda) \in \mathscr{M}_{d}(A)$ be a lifting of $\left(X_{0}, \lambda_{0}\right)$ so we have a commutative diagram of solid arrows

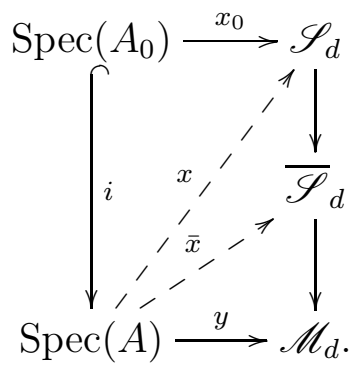

Since $\mathscr{S}_{d}$ is a $\mathbb{G}_{m}$-gerbe over $\overline{\mathscr{S}}_{d}$, the obstruction to lifting a map $\bar{x}$ as indicated to a morphism $x$ is given by a class in $H^{2}(\operatorname{Spec}(A), \widetilde{I})=0$, and therefore any such map $\bar{x}$ can be lifted to a map $x$. Furthermore, the set of isomorphism classes of such liftings $x$ of $\bar{x}$ is given by $H^{1}(\operatorname{Spec}(A), \widetilde{I})=0$ so in fact the lifting $x$ is unique up to isomorphism. The isomorphism is not unique but determined up to the action of

$$
\operatorname{Ker}\left(A^{*} \rightarrow A_{0}^{*}\right) \simeq I .
$$

From this it follows that it suffices to show the following:

(i) The lifting $(X, \lambda)$ of $\left(X_{0}, \lambda_{0}\right)$ can be extended to a lifting $((X, \lambda), Y, P)$ of $\left(\left(X_{0}, \lambda_{0}\right), Y_{0}, P_{0}\right)$.

(ii) This extension $((X, \lambda), Y, P)$ of $(X, \lambda)$ is unique up to isomorphism.

(iii) The automorphisms of the triple $((X, \lambda), Y, P)$ which are the identity on $(X, \lambda)$ and reduce to the identity over $A_{0}$ are all given by scalar multiplication on $P$ by elements of $1+I \subset A^{*}$.

Statement (i) is shown in [15, 6.3].

Next we prove the uniqueness statements in (ii) and (iii). Following the notation of [15, Discussion preceding 5.2], let $s \mathscr{D}_{X / A}$ denote the stack of simple, universally gluable, relatively 
perfect complexes on $X$, and let $s D_{X / A}$ denote its rigidifcation with respect to the $\mathbb{G}_{m}$-action given by scalar multiplication. The complex $P_{0}$ on $X_{0} \times_{A_{0}} Y_{0}$ defines a morphism

$$
Y_{0} \rightarrow s D_{X / A} \otimes_{A} A_{0}
$$

which by [15, 5.2 (ii)] is an open imbedding. Any extension of $(X, \lambda)$ to a lifting $((X, \lambda), Y, P)$ defines an open imbedding $Y \hookrightarrow s D_{X / A}$. This implies that $Y$, viewed as a deformation of $Y_{0}$ for which there exists a lifting $P$ of $P_{0}$ to $X \times_{A} Y$, is unique up to unique isomorphism.

Let $Y$ denote the unique lifting of $Y_{0}$ to an open subspace of $s D_{X / A}$. By [14, 3.1.1 (2)] the set of isomorphism classes of liftings of $P_{0}$ to $X \times_{A} Y$ is a torsor under

$$
\operatorname{Ext}_{X_{k} \times Y_{k}}^{1}\left(P_{k}, P_{k}\right) \otimes I
$$

which is 0 by Lemma 3.7 (ii). From this it follows that $P$ is unique up to isomorphism, and also by Lemma 3.7 (iii) we get the statement that the only infinitesimal automorphisms of the triple $((X, \lambda), Y, P)$ are given by scalar multiplication by elements of $1+I$.

3.10. There is an automorphism

$$
\sigma: \mathscr{S}_{d} \rightarrow \mathscr{S}_{d}
$$

satisfying $\sigma^{2}=\mathrm{id}$. This automorphism is defined by sending a triple $((X, \lambda), Y, P)$ to $\left(\left(Y, \lambda_{Y}\right), X, P^{\vee}[2]\right)$. This automorphism induces an involution $\bar{\sigma}: \overline{\mathscr{S}}_{d} \rightarrow \overline{\mathscr{S}}_{d}$ over the involution $\gamma: \mathscr{M}_{d} \times \mathscr{M}_{d} \rightarrow \mathscr{M}_{d} \times \mathscr{M}_{d}$ switching the factors.

Remark 3.11. In fact the stack $\mathscr{S}_{d}$ is defined over $\mathbb{Z}[1 / d]$ and Theorems [3.6] and 3.9 also hold over $\mathbb{Z}[1 / d]$. In what follows we write $\mathscr{S}_{d, \mathbb{Z}[1 / d]}$ for this stack over $\mathbb{Z}[1 / d]$.

\section{Deformations of autoequivalences}

In this section, we describe the obstructions to deforming Fourier-Mukai equivalences. The requisite technical machinery for this is worked out in [12] and [13]. The results of this section will play a crucial role in Section 6.

Throughout this section let $k$ be a perfect field of positive characteristic $p$ and ring of Witt vectors $W$. For an integer $n$ let $R_{n}$ denote the ring $k[t] /\left(t^{n+1}\right)$, and let $R$ denote the ring $k[[t]]$.

4.1. Let $X_{n+1} / R_{n+1}$ be a smooth proper scheme over $R_{n+1}$ with reduction $X_{n}$ to $R_{n}$. We then have the associated relative Kodaira-Spencer class, defined in [12, p. 486], which is the morphism in $D\left(X_{n}\right)$

$$
\kappa_{X_{n} / X_{n+1}}: \Omega_{X_{n} / R_{n}}^{1} \rightarrow \mathscr{O}_{X_{n}}[1]
$$

defined as the morphism corresponding to the short exact sequence

$$
\left.0 \longrightarrow \mathscr{O}_{X_{n}} \stackrel{\cdot d t}{\longrightarrow} \Omega_{X_{n+1} / k}^{1}\right|_{X_{n}} \longrightarrow \Omega_{X_{n} / R_{n}}^{1} \longrightarrow 0
$$

4.2. We also have the relative universal Atiyah class which is a morphism

$$
\alpha_{n}: \mathscr{O}_{\Delta_{n}} \rightarrow i_{n *} \Omega_{X_{n} / R_{n}}^{1}[1]
$$

in $D\left(X_{n} \times_{R_{n}} X_{n}\right)$, where $i_{n}: X_{n} \rightarrow X_{n} \times_{R_{n}} X_{n}$ is the diagonal morphism and $\mathscr{O}_{\Delta_{n}}$ denotes $i_{n *} \mathscr{O}_{X_{n}}$. 
This map $\alpha_{n}$ is given by the class of the short exact sequence

$$
0 \rightarrow I / I^{2} \rightarrow \mathscr{O}_{X_{n} \times_{R_{n}} X_{n}} / I^{2} \rightarrow \mathscr{O}_{\Delta_{n}} \rightarrow 0
$$

where $I \subset \mathscr{O}_{X_{n} \times_{R_{n}} X_{n}}$ is the ideal of the diagonal. Note that to get the morphism $\alpha_{n}$ we need to make a choice of isomorphism $I / I^{2} \simeq \Omega_{X_{n} / R_{n}}^{1}$, which implies that the relative universal Atiyah class is not invariant under the map switching the factors, but rather changes by -1 .

4.3. Define the relative Hochschild cohomology of $X_{n} / R_{n}$ by

$$
H H^{*}\left(X_{n} / R_{n}\right):=\operatorname{Ext}_{X_{n} \times_{R_{n}} X_{n}}^{*}\left(\mathscr{O}_{\Delta_{n}}, \mathscr{O}_{\Delta_{n}}\right) .
$$

The composition

$$
\mathscr{O}_{\Delta_{n}} \stackrel{\alpha_{n}}{\longrightarrow} i_{n *} \Omega_{X_{n} / R_{n}}^{1}[1] \stackrel{i_{n *} \kappa_{X_{n} / X_{n+1}}}{\longrightarrow} \mathscr{O}_{\Delta_{n}}[2]
$$

is a class

$$
\nu_{X_{n} / X_{n+1}} \in H H^{2}\left(X_{n} / R_{n}\right) .
$$

4.4. Suppose now that $Y_{n} / R_{n}$ is a second smooth proper scheme with a smooth lifting $Y_{n+1} / R_{n+1}$ and that $E_{n} \in D\left(X_{n} \times_{R_{n}} Y_{n}\right)$ is a $R_{n}$-perfect complex.

Consider the class

$$
\nu:=\nu_{X_{n} \times_{R_{n}} Y_{n} / X_{n+1} \times_{R_{n+1}} Y_{n+1}}: \mathscr{O}_{\Delta_{n, X_{n} \times R_{n}} Y_{n}} \rightarrow \mathscr{O}_{\Delta_{n, X_{n} \times R_{n}} Y_{n}}[2] .
$$

Viewing this is a morphism of Fourier-Mukai kernels

$$
D\left(X_{n} \times_{R_{n}} Y_{n}\right) \rightarrow D\left(X_{n} \times_{R_{n}} Y_{n}\right)
$$

and applying it to $E_{n}$ we get a class

$$
\omega\left(E_{n}\right) \in \operatorname{Ext}_{X_{n} \times_{R_{n}} Y_{n}}^{2}\left(E_{n}, E_{n}\right) .
$$

In the case when

$$
\operatorname{Ext}_{X_{0} \times Y_{0}}^{1}\left(E_{0}, E_{0}\right)=0
$$

which will hold in the cases of interest in this paper, we know by [12, Lemma 3.2] that the class $\omega\left(E_{n}\right)$ is 0 if and only if $E_{n}$ lifts to a perfect complex on $X_{n+1} \times_{R_{n+1}} Y_{n+1}$.

4.5. To analyze the class $\omega\left(E_{n}\right)$ it is useful to translate it into a statement about classes in $H H^{2}\left(Y_{n} / R_{n}\right)$. This is done using Toda's argument [26, Proof of 5.6]. Let

$$
E_{n} \circ: D\left(X_{n} \times_{R_{n}} X_{n}\right) \rightarrow D\left(X_{n} \times{ }_{R_{n}} Y_{n}\right)
$$

denote the map sending an object $K \in D\left(X_{n} \times_{R_{n}} X_{n}\right)$ to the complex representing the Fourier-Mukai transform $\Phi_{E_{n}} \circ \Phi_{K}$. Explicitly it is given by the complex

$$
p_{13 *}\left(p_{12}^{*} K \otimes p_{23}^{*} E_{n}\right),
$$

where $p_{i j}$ denote the various projections from $X_{n} \times_{R_{n}} X_{n} \times_{R_{n}} Y_{n}$. As in loc. cit. the diagram

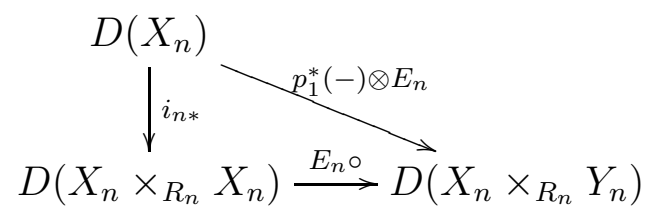

commutes. 
In particular we get a morphism

$$
\eta_{X}^{*}: H H^{*}\left(X_{n} / R_{n}\right) \rightarrow \operatorname{Ext}_{X_{n} \times_{R_{n}} Y_{n}}^{*}\left(E_{n}, E_{n}\right) .
$$

Now assume that both $X_{n}$ and $Y_{n}$ have relative dimension $d$ over $R_{n}$ and that the relative canonical sheaves of $X_{n}$ and $Y_{n}$ over $R_{n}$ are trivial. Let $E_{n}^{\vee} \operatorname{denote} \mathscr{R} \operatorname{Hom}\left(E_{n}, \mathscr{O}_{X_{n} \times_{R_{n}} Y_{n}}\right)$ viewed as an object of $D\left(Y_{n} \times_{R_{n}} X_{n}\right)$. In this case the functor

$$
\Phi_{E_{n}^{\vee}[d]}: D\left(Y_{n}\right) \rightarrow D\left(X_{n}\right)
$$

is both a right and left adjoint of $\Phi_{E_{n}}$ [4, 4.5]. By the same argument, the functor

$$
\circ E_{n}^{\vee}[d]: D\left(X_{n} \times_{R_{n}} Y_{n}\right) \rightarrow D\left(Y_{n} \times_{R_{n}} Y_{n}\right),
$$

defined in the same manner as $E_{n} \circ$ has left and right adjoint given by

$$
\circ E_{n}: D\left(Y_{n} \times_{R_{n}} Y_{n}\right) \rightarrow D\left(X_{n} \times_{R_{n}} Y_{n}\right) .
$$

Composing with the adjunction maps

$$
\alpha: \mathrm{id} \rightarrow \circ E_{n} \circ E_{n}^{\vee}[d], \quad \beta: \circ E_{n} \circ E_{n}^{\vee}[d] \rightarrow \text { id }
$$

applied to the diagonal $\mathscr{O}_{\Delta_{Y_{n}}}$ we get a morphism

$$
\eta_{Y *}: \operatorname{Ext}_{X_{n} \times_{R_{n}} Y_{n}}^{*}\left(E_{n}, E_{n}\right) \rightarrow H H^{*}\left(Y_{n} / R_{n}\right) .
$$

We denote the composition

$$
\eta_{Y *} \eta_{X}^{*}: H H^{*}\left(X_{n} / R_{n}\right) \rightarrow H H^{*}\left(Y_{n} / R_{n}\right)
$$

by $\Phi_{E_{n}}^{H H^{*}}$. In the case when $E_{n}$ defines a Fourier-Mukai equivalence this agrees with the standard definition (see for example [26]).

4.6. Evaluating the adjunction maps (4.5.1) on $\mathscr{O}_{\Delta_{Y_{n}}}$ we get a morphism

$$
\mathscr{O}_{\Delta_{Y_{n}}} \stackrel{\alpha}{\longrightarrow} \mathscr{O}_{\Delta_{Y_{n}}} \circ E_{n} \circ E_{n}^{\vee}[d] \stackrel{\beta}{\longrightarrow} \mathscr{O}_{\Delta_{Y_{n}}}
$$

We say that $E_{n}$ is admissible if this composition is the identity map.

If $E_{n}$ is a Fourier-Mukai equivalence then it is clear that $E_{n}$ is admissible. Another example is if there exists a lifting $(\mathscr{X}, \mathscr{Y}, \mathscr{E})$ of $\left(X_{n}, Y_{n}, E_{n}\right)$ to $R$, where $\mathscr{X}$ and $\mathscr{Y}$ are smooth proper $R$-schemes with trivial relative canonical bundles and $\mathscr{E}$ is a $R$-perfect complex on $\mathscr{X} \times_{R} \mathscr{Y}$, such that the restriction $\mathscr{E}$ to the generic fiber defines a Fourier-Mukai equivalence. Indeed in this case the map (4.6.1) is the reduction of the corresponding map $\mathscr{O}_{\Delta \mathscr{Y}} \rightarrow \mathscr{O}_{\Delta_{\mathscr{Y}}}$ defined over $R$, which in turn is determined by its restriction to the generic fiber.

4.7. Consider Hochschild homology

$$
H H_{i}\left(X_{n} / R_{n}\right):=H^{-i}\left(X_{n}, L i_{n}^{*} \mathscr{O}_{\Delta_{n}}\right)
$$

By the argument of [5, §5] we also get an action

$$
\Phi_{E_{n}}^{H H_{*}}: H H_{*}\left(X_{n} / R_{n}\right) \rightarrow H H_{*}\left(Y_{n} / R_{n}\right) .
$$


Hochschild homology is a module over Hochschild cohomology, and an exercise (that we do not write out here) shows that the following diagram

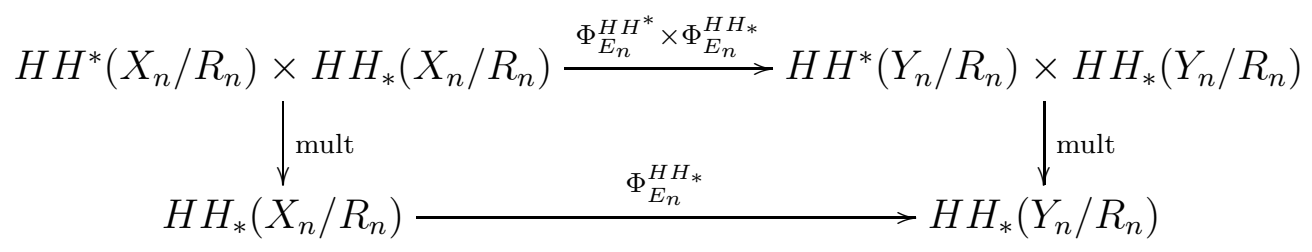

commutes.

4.8. Using this we can describe the obstruction $\omega\left(E_{n}\right)$ in a different way, assuming that $E_{n}$ is admissible. First note that viewing the relative Atiyah class of $X_{n} \times_{R_{n}} Y_{n}$ as a morphism of Fourier-Mukai kernels we get the Atiyah class of $E_{n}$ which is a morphism

$$
A\left(E_{n}\right): E_{n} \rightarrow E_{n} \otimes \Omega_{X_{n} \times_{R_{n}} Y_{n} / R_{n}}^{1}[1]
$$

in $D\left(X_{n} \times_{R_{n}} Y_{n}\right)$. There is a natural decomposition

$$
\Omega_{X_{n} \times_{R_{n}} Y_{n} / R_{n}}^{1} \simeq p_{1}^{*} \Omega_{X_{n} / R_{n}}^{1} \oplus p_{2}^{*} \Omega_{Y_{n} / R_{n}}^{1},
$$

so we can write $A\left(E_{n}\right)$ as a sum of two maps

$$
A\left(E_{n}\right)_{X}: E_{n} \rightarrow E_{n} \otimes p_{1}^{*} \Omega_{X_{n} / R_{n}}^{1}[1], \quad A\left(E_{n}\right)_{Y}: E_{n} \rightarrow E_{n} \otimes p_{2}^{*} \Omega_{X_{n} / R_{n}}^{1}[1] .
$$

Similarly the Kodaira-Spencer class of $X_{n} \times_{R_{n}} Y_{n}$ can be written as the sum of the two pullbacks

$$
p_{1}^{*} \kappa_{X_{n} / X_{n+1}}: p_{1}^{*} \Omega_{X_{n} / R_{n}}^{1} \rightarrow p_{1}^{*} \mathscr{O}_{X_{n}}[1], \quad p_{2}^{*} \kappa_{Y_{n} / Y_{n+1}}: p_{2}^{*} \Omega_{Y_{n} / R_{n}}^{1} \rightarrow p_{2}^{*} \mathscr{O}_{Y_{n}}[1] .
$$

It follows that the obstruction $\omega\left(E_{n}\right)$ can be written as a sum

$$
\omega\left(E_{n}\right)=\left(p_{1}^{*} \kappa_{X_{n} / X_{n+1}} \circ A\left(E_{n}\right)_{X}\right)+\left(p_{2}^{*} \kappa\left(Y_{n} / Y_{n+1}\right) \circ A\left(E_{n}\right)_{Y}\right) .
$$

Now by construction we have

$$
\eta_{X_{n}}^{*}\left(\nu_{X_{n} / X_{n+1}}\right)=p_{1}^{*} \kappa_{X_{n} / X_{n+1}} \circ A\left(E_{n}\right)_{X}
$$

and

$$
\eta_{Y_{n} *}\left(p_{2}^{*} \kappa\left(Y_{n} / Y_{n+1}\right) \circ A\left(E_{n}\right)_{Y}\right)=-\nu_{Y_{n} / Y_{n+1}}
$$

the sign coming from the asymmetry in the definition of the relative Atiyah class (it is in the verification of this second formula that we use the assumption that $E_{n}$ is admissible). Summarizing we find the formula

$$
\eta_{Y_{n} *}\left(\omega\left(E_{n}\right)\right)=\Phi_{E_{n}}^{H H^{*}}\left(\nu_{X_{n} / X_{n+1}}\right)-\nu_{Y_{n} / Y_{n+1}}
$$

In the case when $\Phi_{E_{n}}$ is an equivalence the maps $\eta_{Y_{n} *}$ and $\eta_{X_{n}}^{*}$ are isomorphisms, so the obstruction $\omega\left(E_{n}\right)$ vanishes if and only if we have

$$
\Phi_{E_{n}}^{H H^{*}}\left(\nu_{X_{n} / X_{n+1}}\right)-\nu_{Y_{n} / Y_{n+1}}=0 .
$$

Remark 4.9. By [12, Remark 2.3 (iii)], the functor $\Phi_{E_{n}}$ is an equivalence if and only if $\Phi_{E_{0}}: D\left(X_{0}\right) \rightarrow D\left(Y_{0}\right)$ is an equivalence.

Corollary 4.10. Suppose $F_{n} \in D\left(X_{n} \times_{R_{n}} Y_{n}\right)$ defines a Fourier-Mukai equivalence, and that $E_{n} \in D\left(X_{n} \times_{R_{n}} Y_{n}\right)$ is another admissible $R_{n}$-perfect complex such that $\Phi_{F_{n}}^{H H^{*}}=\Phi_{E_{n}}^{H^{*}}$. If $E_{n}$ lifts to a $R_{n+1}$-perfect complex $E_{n+1} \in D\left(X_{n+1} \times_{R_{n+1}} Y_{n+1}\right)$ then so does $F_{n}$. 
Proof. Indeed the condition that $\Phi_{F_{n}}^{H H^{*}}=\Phi_{E_{n}}^{H H^{*}}$ ensures that

$$
\eta_{Y_{n} *}\left(\omega\left(E_{n}\right)\right)=\eta_{Y_{n} *}\left(\omega\left(F_{n}\right)\right),
$$

and since $\omega\left(E_{n}\right)=0$ we conclude that $\omega\left(F_{n}\right)=0$.

4.11. The next step is to understand the relationship between $\Phi_{E_{n}}^{H H^{*}}$ and the action of $\Phi_{E_{n}}$ on the cohomological realizations of the Mukai motive.

Assuming that the characteristic $p$ is bigger than the dimension of $X_{0}$ (which in our case will be a K3 surface so we just need $p>2$ ) we can exponentiate the relative Atiyah class to get a map

$$
\exp \left(\alpha_{n}\right): \mathscr{O}_{\Delta_{n}} \rightarrow \oplus_{i} i_{n *} \Omega_{X_{n} / R_{n}}^{i}
$$

which by adjunction defines a morphism

$$
L i_{n}^{*} \mathscr{O}_{\Delta_{n}} \rightarrow \oplus_{i} \Omega_{X_{n} / R_{n}}^{i}
$$

in $D\left(X_{n}\right)$. By [1, Theorem 0.7], which also holds in positive characteristic subject to the bounds on dimension, this map is an isomorphism. We therefore get an isomorphism

$$
I^{H K R}: H H^{*}\left(X_{n} / R_{n}\right) \rightarrow H T^{*}\left(X_{n} / R_{n}\right),
$$

where we write

$$
H T^{*}\left(X_{n} / R_{n}\right):=\oplus_{p+q=*} H^{p}\left(X_{n}, \bigwedge^{q} T_{X_{n} / R_{n}}\right)
$$

We write

$$
I_{X_{n}}^{K}: H H^{*}\left(X_{n} / R_{n}\right) \rightarrow H T^{*}\left(X_{n} / R_{n}\right)
$$

for the composition of $I^{H K R}$ with multiplication by the inverse square root of the Todd class of $X_{n} / R_{n}$, as in [6, 1.7].

The isomorphism (4.11.1) also defines an isomorphism

$$
I_{H K R}: H H_{*}\left(X_{n} / R_{n}\right) \rightarrow H \Omega_{*}\left(X_{n} / R_{n}\right),
$$

where

$$
H \Omega_{*}\left(X_{n} / R_{n}\right):=\oplus_{q-p=*} H^{p}\left(X_{n}, \Omega_{X_{n} / R_{n}}^{q}\right) .
$$

We write

$$
I_{K}^{X_{n}}: H H_{*}\left(X_{n} / R_{n}\right) \rightarrow H \Omega_{*}\left(X_{n} / R_{n}\right)
$$

for the composition of $I_{H K R}$ with multiplication by the square root of the Todd class of $X_{n} / R_{n}$.

We will consider the following condition $(\star)$ on a $R_{n}$-perfect complex $E_{n} \in D\left(X_{n} \times_{R_{n}} Y_{n}\right)$ :

( ) The diagram

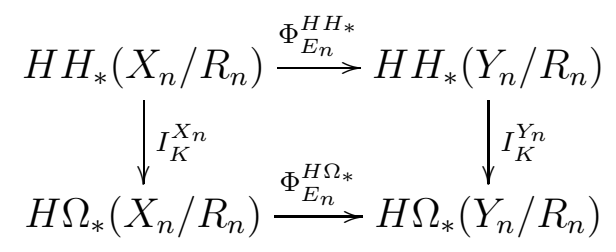

commutes. 
Remark 4.12. We expect this condition to hold in general. Over a field of characteristic 0 this is shown in [21, 1.2]. We expect that a careful analysis of denominators occurring of their proof will verify $(\star)$ quite generally with some conditions on the characteristic relative to the dimension of the schemes. However, we will not discuss this further in this paper.

4.13. There are two cases we will consider in this paper were $(\star)$ is known to hold:

(i) If $E_{n}=\mathscr{O}_{\Gamma_{n}}$ is the structure sheaf of the graph of an isomorphism $\gamma_{n}: X_{n} \rightarrow Y_{n}$. In this case the induced maps on Hochschild cohomology and $H \Omega_{*}$ are simply given by pushforward $\gamma_{n *}$ and condition $(\star)$ immediately holds.

(ii) Suppose $B \rightarrow R_{n}$ is a morphism from an integral domain $B$ which is flat over $W$ and that there exists a lifting $(\mathscr{X}, \mathscr{Y}, \mathscr{E})$ of $\left(X_{n}, Y_{n}, E_{n}\right)$ to $B$, where $\mathscr{X}$ and $\mathscr{Y}$ are proper and smooth over $B$ and $\mathscr{E} \in D\left(\mathscr{X} \times{ }_{B} \mathscr{Y}\right)$ is a $B$-perfect complex pulling back to $E_{n}$. Suppose further that the groups $H H^{*}(\mathscr{X} / B)$ and $H H^{*}(\mathscr{Y} / B)$ are flat over $B$ and their formation commutes with base change (this holds for example if $\mathscr{X}$ and $\mathscr{Y}$ are K3 surfaces). Then $(\star)$ holds. Indeed it suffices to verify the commutativity of the corresponding diagram over $B$, and this in turn can be verified after passing to the field of fractions of $B$. In this case the result holds by [21, 1.2].

Lemma 4.14. Let $E_{n}, F_{n} \in D\left(X_{n} \times_{R_{n}} Y_{n}\right)$ be two $R_{n}$-perfect complexes satisfying condition $(\star)$. Suppose further that the maps $\Phi_{E_{0}}^{\text {crys }}$ and $\Phi_{F_{0}}^{\text {crys }}$ on the crystalline realizations $\widetilde{H}\left(X_{0} / W\right) \rightarrow$ $\widetilde{H}\left(Y_{0} / W\right)$ of the Mukai motive are equal. Then the maps $\Phi_{E_{n}}^{H H_{*}}$ and $\Phi_{F_{n}}^{H H_{*}}$ are also equal. Furthermore if the maps on the crystalline realizations are isomorphisms then $\Phi_{E_{n}}^{H_{*}{ }_{*}}$ and $\Phi_{F_{n}}^{H H_{*}}$ are also isomorphisms.

Proof. Since $H \Omega_{*}\left(X_{n} / R_{n}\right)$ (resp. $H \Omega_{*}\left(Y_{n} / R_{n}\right)$ ) is obtained from the de Rham realization $\widetilde{H}_{\mathrm{dR}}\left(X_{n} / R_{n}\right)$ (resp. $\widetilde{H}_{\mathrm{dR}}\left(X_{n} / R_{n}\right)$ ) of the Mukai motive of $X_{n} / R_{n}$ (resp. $Y_{n} / R_{n}$ ) by passing to the associated graded, it suffices to show that the two maps

$$
\Phi_{E_{n}}^{\mathrm{dR}}, \Phi_{F_{n}}^{\mathrm{dR}}: \widetilde{H}_{\mathrm{dR}}\left(X_{n} / R_{n}\right) \rightarrow \widetilde{H}_{\mathrm{dR}}\left(Y_{n} / R_{n}\right)
$$

are equal, and isomorphisms when the crystalline realizations are isomorphisms. By the comparison between crystalline and de Rham cohomology it suffices in turn to show that the two maps on the crystalline realizations

$$
\Phi_{E_{n}}^{\text {crys }}, \Phi_{F_{n}}^{\text {crys }}: \widetilde{H}_{\text {crys }}\left(X_{n} / W[t] /\left(t^{n+1}\right)\right) \rightarrow \widetilde{H}_{\text {crys }}\left(Y_{n} / W[t] /\left(t^{n+1}\right)\right)
$$

are equal. Via the Berthelot-Ogus isomorphism [3, 2.2], which is compatible with Chern classes, these maps are identified after tensoring with $\mathbb{Q}$ with the two maps obtained by base change from

The result follows.

$$
\Phi_{E_{0}}^{\text {crys }}, \Phi_{F_{0}}^{\text {crys }}: \widetilde{H}_{\text {crys }}\left(X_{0} / W\right) \rightarrow \widetilde{H}_{\text {crys }}\left(Y_{0} / W\right)
$$

4.15. In the case when $X_{n}$ and $Y_{n}$ are K3 surfaces the action of $H H^{*}\left(X_{n} / R_{n}\right)$ on $H H_{*}\left(X_{n} / R_{n}\right)$ is faithful. Therefore from Lemma 4.14 we obtain the following.

Corollary 4.16. Assume that $X_{n}$ and $Y_{n}$ are K3 surfaces and that $E_{n}, F_{n} \in D\left(X_{n} \times_{R_{n}} Y_{n}\right)$ are two $R_{n}$-perfect complexes satisfying condition $(\star)$. Suppose further that $\Phi_{E_{0}}^{\text {crys }}$ and $\Phi_{F_{0}}^{\text {crys }}$ are equal on the crystalline realizations of the Mukai motives of the reductions. Then $\Phi_{E_{n}}^{H H^{*}}$ and $\Phi_{F_{n}}^{H H^{*}}$ are equal. 
Proof. Indeed since homology is a faithful module over cohomology the maps $\Phi_{E_{n}}^{H H^{*}}$ and $\Phi_{F_{n}}^{H H^{*}}$ are determined by the maps on Hochschild homology which are equal by Lemma 4.14 .

Corollary 4.17. Let $X_{n+1}$ and $Y_{n+1}$ be K3 surfaces over $R_{n+1}$ and assume given an admissible $R_{n+1}$-perfect complex $E_{n+1}$ on $X_{n+1} \times_{R_{n+1}} Y_{n+1}$ such that $E_{n}$ satisfies condition $(\star)$. Assume given an isomorphism $\sigma_{n}: X_{n} \rightarrow Y_{n}$ over $R_{n}$ such that the induced map $\sigma_{0}: X_{0} \rightarrow Y_{0}$ defines the same map on crystalline realizations of the Mukai motive as $E_{0}$. Then $\sigma_{n}$ lifts to an isomorphism $\sigma_{n+1}: X_{n+1} \rightarrow Y_{n+1}$.

Proof. Indeed by (4.8.1) and the fact that $\Phi_{E_{n}}^{H H^{*}}$ and $\Phi_{\Gamma_{\sigma_{n}}}^{H H^{*}}$ are equal by Corollary 4.16, we see that the obstruction to lifting $\sigma_{n}$ is equal to the obstruction to lifting $E_{n}$, which is zero by assumption.

\section{A REMARK ON REDUCTION TYPES}

5.1. In the proof of Theorem 1.2 we need the following Theorem 5.3, whose proof relies on known characteristic 0 results obtained from Hodge theory. In Section 10 below we give a different algebraic argument for Theorem 5.3 in a special case which suffices for the proof of Theorem 1.2,

5.2. Let $V$ be a complete discrete valuation ring with field of fractions $K$ and residue field $k$. Let $X / V$ be a projective K3 surface with generic fiber $X_{K}$, and let $Y_{K}$ be a second K3 surface over $K$ such that the geometric fibers $X_{\bar{K}}$ and $Y_{\bar{K}}$ are derived equivalent.

Theorem 5.3. Under these assumptions the K3 surface $Y_{K}$ has potentially good reduction.

Remark 5.4. Here potentially good reduction means that after possibly replacing $V$ be a finite extension there exists a $\mathrm{K} 3$ surface $Y / V$ whose generic fiber is $Y_{K}$.

Proof of Theorem [5.3. We use [15, 1.1 (1)] which implies that after replacing $V$ by a finite extension $Y_{K}$ is isomorphic to a moduli space of sheaves on $X_{K}$.

After replacing $V$ by a finite extension we may assume that we have a complex $P \in$ $D(X \times Y)$ defining an equivalence

$$
\Phi_{P}: D\left(X_{\bar{K}}\right) \rightarrow D\left(Y_{\bar{K}}\right)
$$

Let $E \in D(Y \times X)$ be the complex defining the inverse equivalence

$$
\Phi_{E}: D\left(Y_{\bar{K}}\right) \rightarrow D\left(X_{\bar{K}}\right)
$$

to $\Phi_{P}$. Let $\nu:=\Phi_{E}(0,0,1) \in A^{*}\left(X_{\bar{K}}\right)_{\text {num, } \mathbb{Q}}$ be the Mukai vector of a fiber of $E$ at a closed point $y \in Y_{\bar{K}}$ and write

$$
\nu=\left(r,\left[L_{X}\right], s\right) \in A^{0}\left(X_{\bar{K}}\right)_{\text {num }, \mathbb{Q}} \oplus A^{1}\left(X_{\bar{K}}\right)_{\text {num }, \mathbb{Q}} \oplus A^{2}\left(X_{\bar{K}}\right)_{\text {num }, \mathbb{Q}} .
$$

By [15, 8.1] we may after possible changing our choice of $P$, which may involve another extension of $V$, assume that $r$ is prime to $p$ and that $L_{X}$ is very ample. Making another extension of $V$ we may assume that $\nu$ is defined over $K$, and therefore by specialization also defines an element, which we denote by the same letter,

$$
\nu=\left(r,\left[L_{X}\right], s\right) \in \mathbb{Z} \oplus \operatorname{Pic}(X) \oplus \mathbb{Z} .
$$


This class has the property that $r$ is prime to $p$ and that there exists another class $\nu^{\prime}$ such that $\left\langle\nu, \nu^{\prime}\right\rangle=1$. This implies in particular that $\nu$ restricts to a primitive class on the closed fiber. Fix an ample class $h$ on $X$, and let $\mathscr{M}_{h}(\nu)$ denote the moduli space of semistable sheaves on $X$ with Mukai vector $\nu$. By [15, 3.16] the stack $\mathscr{M}_{h}(\nu)$ is a $\mu_{r}$-gerbe over a relative K3 surface $M_{h}(\nu) / V$, and by [15, 8.2] we have $Y_{\bar{K}} \simeq M_{h}(\nu)_{\bar{K}}$. In particular, $Y$ has potentially good reduction.

Remark 5.5. As discussed in [17, p. 2], to obtain Theorem [5.3 it suffices to know that every $\mathrm{K} 3$ surface $Z_{K}$ over $K$ has potentially semistable reduction and this would follow from standard conjectures on resolution of singularities and toroidization of morphisms in mixed and positive characteristic. In the setting of Theorem 5.3, once we know that $Y_{K}$ has potentially semistable reduction then by [17, Theorem on bottom of p. 2] we obtain that $Y_{K}$ has good reduction since the Galois representation $H^{2}\left(Y_{\bar{K}}, \mathbb{Q}_{\ell}\right)$ is unramified being isomorphic to direct summand of the $\ell$-adic realization $\widetilde{H}\left(X_{\bar{K}}, \mathbb{Q}_{\ell}\right)$ of the Mukai motive of $X_{K}$.

5.6. One can also consider the problem of extending $Y_{K}$ over a higher dimensional base. Let $B$ denote a normal finite type $k$-scheme with a point $s \in B(k)$ and let $X / B$ be a projective family of K3 surfaces. Let $K$ be the function field of $B$ and let $Y_{K}$ be a second $\mathrm{K} 3$ surface over $K$ Fourier-Mukai equivalent to $X_{K}$. Dominating $\mathscr{O}_{B, s}$ by a suitable complete discrete valuation ring $V$ we can find a morphism

$$
\rho: \operatorname{Spec}(V) \rightarrow B
$$

sending the closed point of $\operatorname{Spec}(V)$ to $s$ and an extension $Y_{V}$ of $\rho^{*} Y_{K}$ to a smooth projective K3 surface over $V$. In particular, after replacing $B$ by its normalization in a finite extension of $K$ we can find a primitive polarization $\lambda_{K}$ on $Y_{K}$ of degree prime to the characteristic such that $\rho^{*} \lambda_{K}$ extends to a polarization on $Y_{V}$. We then have a commutative diagram of solid arrows

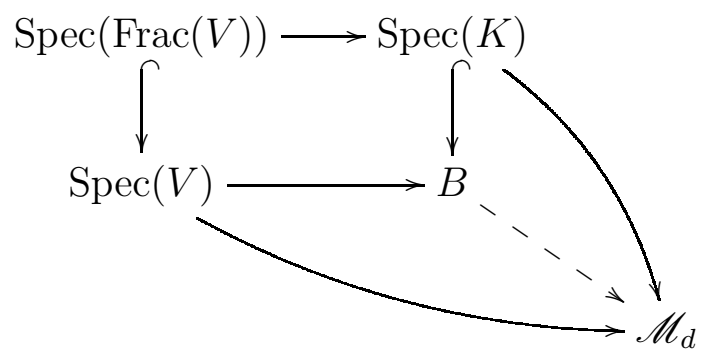

for a suitable integer $d$. Base changing to a suitable étale neighborhood $U \rightarrow \mathscr{M}_{d}$ of the image of the closed point of $\operatorname{Spec}(V)$, with $U$ an affine scheme, we can after shrinking and possibly replacing $B$ by an alteration find a commutative diagram

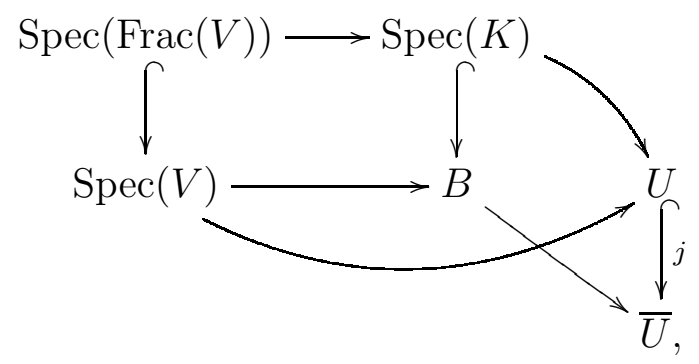


where $j$ is a dense open imbedding and $\bar{U}$ is projective over $k$. It follows that the image of $s$ in $\bar{U}$ in fact lands in $U$ which gives an extension of $Y_{K}$ to a neighborhood of $s$. This discussion implies the following:

Corollary 5.7. In the setup of Proposition 5.6, we can, after replacing $(B, s)$ by a neighborhood of a point in the preimage of $s$ in an alteration of $B$, find an extension of $Y_{K}$ to a K3 surface over $B$.

\section{Supersingular Reduction}

6.1. Let $B$ be a normal scheme of finite type over an algebraically closed field $k$ of odd positive characteristic $p$. Let $K$ denote the function field of $B$ and let $s \in B$ be a closed point. Let $f: X \rightarrow B$ be a projective K3 surface over $B$ and let $Y_{K} / K$ be a second K3 surface over $K$ such that there exists a strongly filtered Fourier-Mukai equivalence

$$
\Phi_{P}: D\left(X_{K}\right) \rightarrow D\left(Y_{K}\right)
$$

defined by an object $P \in D\left(X_{K} \times_{K} Y_{K}\right)$. Assume further that the fiber $X_{s}$ of $X$ over $s$ is a supersingular K3 surface.

6.2. Using Corollary 5.7 we can, after possibly replacing $B$ by a neighborhood of a point over $s$ in an alteration, assume that we have a smooth K3 surface $Y / B$ extending $Y_{K}$ and an extension of the complex $P$ to a $B$-perfect complex $\mathscr{P}$ on $X \times_{B} Y$, and furthermore that the complex $Q$ defining the inverse of $\Phi_{P}$ also extends to a complex $\mathscr{Q}$ on $X \times_{B} Y$. Let $f_{\mathscr{X}}: \mathscr{X} \rightarrow B$ (resp. $f_{\mathscr{Y}}: \mathscr{Y} \rightarrow B$ ) be the structure morphism, and let $\mathscr{H}_{\text {crys }}^{i}(X / B)$ (resp. $\left.\mathscr{H}_{\text {crys }}^{i}(Y / B)\right)$ denote the $F$-crystal $R^{i} f_{\mathscr{X} *} \mathscr{O}_{\mathscr{X} / W}$ (resp. $\left.R^{i} f_{\mathscr{Y} *} \mathscr{O}_{\mathscr{Y} / W}\right)$ on $B / W$ obtained by forming the $i$-th higher direct image of the structure sheaf on the crystalline site of $\mathscr{X} / W$ (resp. $\mathscr{Y} / W$ ). Because $\Phi_{\mathscr{P}}$ is strongly filtered, it induces an isomorphism of $F$-crystals

$$
\Phi_{\mathscr{P}}^{\text {crys }, i}: \mathscr{H}_{\text {crys }}^{i}(X / B) \rightarrow \mathscr{H}_{\text {crys }}^{i}(Y / B)
$$

for all $i$, with inverse defined by $\Phi_{\mathscr{Q}}$. Note that since we are working here with K3 surfaces these morphisms are defined integrally.

We also have the de Rham realizations $\mathscr{H}_{\mathrm{dR}}^{i}(X / B)$ and $\mathscr{H}_{\mathrm{dR}}^{i}(Y / B)$ which are filtered modules with integrable connection on $B$ equipped with filtered isomorphisms compatible with the connections

$$
\Phi_{\mathscr{P}}^{\mathrm{dR}, i}: \mathscr{H}_{\mathrm{dR}}^{i}(X / B) \rightarrow \mathscr{H}_{\mathrm{dR}}^{i}(Y / B)
$$

as well as étale realizations $\mathscr{H}_{\text {ét }}^{i}(X / B)$ and $\mathscr{H}_{\text {ét }}^{i}(Y / B)$ equipped with isomorphisms

$$
\Phi_{\mathscr{P}}^{\text {ét } i}: \mathscr{H}_{\text {ét }}^{i}(X / B) \rightarrow \mathscr{H}_{\text {ét }}^{i}(Y / B) \text {. }
$$

6.3. Let $H_{\text {crys }}^{i}\left(X_{s} / W\right)$ (resp. $H_{\text {crys }}^{i}\left(Y_{s} / W\right)$ ) denote the crystalline cohomology of the fibers over $s$. The isomorphism $\Phi_{\mathscr{P}}^{\text {crys, } i}$ induces an isomorphism

$$
\theta^{i}: H_{\text {crys }}^{i}\left(X_{s} / W\right) \rightarrow H_{\text {crys }}^{i}\left(Y_{s} / W\right)
$$

of $F$-crystals. By [24, Theorem I] this implies that $X_{s}$ and $Y_{s}$ are isomorphic. However, we may not necessarily have an isomorphism which induces $\theta^{2}$ on cohomology. 
6.4. Recall that as discussed in [11, 10.9 (iii)] if $C \subset X_{K}$ is a (-2)-curve then we can perform a spherical twist

$$
T_{\mathscr{O}_{C}}: D\left(X_{K}\right) \rightarrow D\left(X_{K}\right)
$$

whose action on $N S\left(X_{K}\right)$ is the reflection

$$
r_{C}(a):=a+\langle a, C\rangle C .
$$

Proposition 6.5. After possibly changing our choice of model $Y$ for $Y_{K}$, replacing $(B, s)$ by a neighborhood of a point in an alteration over $s$, and composing with a sequence of spherical twists $T_{\mathscr{O}_{C}}$ along $(-2)$-curves in the generic fiber $Y_{K}$, there exists an isomorphism $\sigma: X_{s} \rightarrow Y_{s}$ inducing the isomorphism $\theta^{2}$ on the second crystalline cohomology group. If $\theta^{2}$ preserves the ample cone of the generic fiber then we can find an isomorphism $\sigma$ inducing $\theta^{2}$.

Proof. By [25, 4.4 and 4.5] there exists an isomorphism $\theta_{0}: N S\left(X_{s}\right) \rightarrow N S\left(Y_{s}\right)$ compatible with $\theta^{2}$ in the sense that the diagram

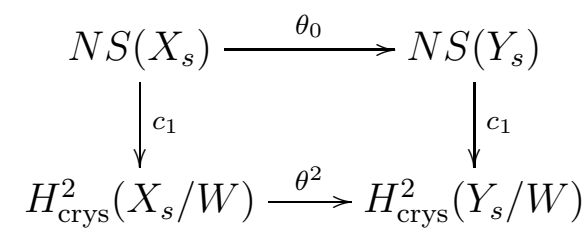

commutes. Note that as discussed in [16, 4.8] the map $\theta_{0}$ determines $\theta^{2}$ by the Tate conjecture for K3 surfaces, proven by Charles, Maulik, and Pera [7, 19, 22]. In particular, if we have an isomorphism $\sigma: X_{s} \rightarrow Y_{s}$ inducing $\pm \theta_{0}$ on Néron-Severi groups then $\sigma$ also induces $\pm \theta^{2}$ on crystalline cohomology. We therefore have to study the problem of finding an isomorphism $\sigma$ compatible with $\theta_{0}$.

Ogus shows in [24, Theorem II] that there exists such an isomorphism $\sigma$ if and only if the map $\theta_{0}$ takes the ample cone to the ample cone. So our problem is to choose a model of $Y$ in such a way that $\pm \theta_{0}$ preserves ample cones. Set

$$
V_{X_{s}}:=\left\{x \in N S\left(X_{s}\right)_{\mathbb{R}} \mid x^{2}>0 \text { and }\langle x, \delta\rangle \neq 0 \text { for all } \delta \in N S\left(X_{s}\right) \text { with } \delta^{2}=-2\right\},
$$

and define $V_{Y_{s}}$ similarly. Being an isometry the map $\theta_{0}$ then induces an isomorphism $V_{X_{s}} \rightarrow$ $V_{Y_{s}}$, which we again denote by $\theta_{0}$. Let $R_{Y_{s}}$ denote the group of automorphisms of $V_{Y_{s}}$ generated by reflections in (-2)-curves and multiplication by -1. By 24, Proposition 1.10 and Remark 1.10.9] the ample cone of $Y_{s}$ is a connected component of $V_{Y_{s}}$ and the group $R_{Y_{s}}$ acts simply transitively on the set of connected components of $V_{Y_{s}}$.

Let us show how to change model to account for reflections by $(-2)$-curves in $Y_{s}$. We show that after replacing $(P, Y)$ by a new pair $\left(P^{\prime}, Y^{\prime}\right)$ consisting of the complex $P \in D\left(X_{K} \times_{K} Y_{K}\right)$ obtained by composing $\Phi_{P}$ with a sequence of spherical twists along (-2)-curves in $Y_{K}$ and replacing $Y$ by a new model $Y^{\prime}$ there exists an isomorphism $\gamma: Y_{s}^{\prime} \rightarrow Y_{s}$ such that the composition

$$
N S\left(X_{s}\right) \stackrel{\theta_{0}}{\longrightarrow} N S\left(Y_{s}\right) \stackrel{r_{C}}{\longrightarrow} N S\left(Y_{s}\right) \stackrel{\gamma^{*}}{\longrightarrow} N S\left(Y_{s}^{\prime}\right)
$$

is equal to the map $\theta_{0}^{\prime}$ defined as for $\theta_{0}$ but using the model $Y^{\prime}$.

Let $C \subset Y_{s}$ be a $(-2)$-curve, and let

$$
r_{C}: N S\left(Y_{s}\right) \rightarrow N S\left(Y_{s}\right)
$$


be the reflection in the (-2)-curve. If $C$ lifts to a curve in the family $Y$ we get a (-2)-curve in the generic fiber and so by replacing our $P$ by the complex $P^{\prime}$ obtained by composition with the spherical twist by this curve in $Y_{K}$ (see [11, 10.9 (iii)]) and setting $Y^{\prime}=Y$ we get the desired new pair. If $C$ does not lift to $Y$, then we take $P^{\prime}=P$ but now replace $Y$ by the flop of $Y$ along $C$ as explained in [24, 2.8].

Thus after making a sequence of replacements $(P, Y) \mapsto\left(P^{\prime}, Y^{\prime}\right)$ we can arrange that $\theta_{0}$ sends the ample cone of $X_{s}$ to plus or minus the ample cone of $Y_{s}$, and therefore we get our desired isomorphism $\sigma$.

To see the last statement, note that we have modified the generic fiber by composing with reflections along (-2)-curves. Therefore if $\lambda$ is an ample class on $X$ with restriction $\lambda_{K}$ to $X_{K}$, and for a general ample divisor $H$ we have $\left\langle\Phi_{P}(\lambda), H\right\rangle>0$, then the same holds on the closed fiber. This implies that the ample cone of $X_{s}$ gets sent to the ample cone of $Y_{s}$ and not its negative.

Remark 6.6. One can also consider étale or de Rham cohomology in Theorem 6.5. Assume we have applied suitable spherical twists and chosen a model $Y$ such that we have an isomorphism $\sigma: X_{s} \rightarrow Y_{s}$ inducing $\pm \theta_{0}$. We claim that the maps

$$
\theta_{\mathrm{dR}}: H_{\mathrm{dR}}^{i}\left(X_{s} / k\right) \rightarrow H_{\mathrm{dR}}^{i}\left(Y_{s} / k\right), \quad \theta_{\text {ét }}: H_{\text {ét }}^{i}\left(X_{s}, \mathbb{Q}_{\ell}\right) \rightarrow H_{\text {ét }}^{i}\left(Y_{s}, \mathbb{Q}_{\ell}\right)
$$

induced by the maps (6.2.1) and (6.2.2) also agree with the maps defined by $\pm \sigma$. For de Rham cohomology this is clear using the comparison with crystalline cohomology, and for the étale cohomology it follows from compatibility with the cycle class map.

6.7. With notation as assumptions as in Theorem 6.5 assume further that $B$ is a curve or a complete discrete valuation ring, and that we have chosen a model $Y$ such that each of the reductions satisfies condition $(\star)$ and such that the map $\theta_{0}$ in (6.5.1) preserves plus or minus the ample cones. Let $\sigma: X_{s} \rightarrow Y_{s}$ be an isomorphism inducing $\pm \theta_{0}$.

Lemma 6.8. The isomorphism $\sigma$ lifts to an isomorphism $\tilde{\sigma}: X \rightarrow Y$ over the completion $\widehat{B}$ at $s$ inducing the maps defined by $\pm \Phi_{\mathscr{P}}^{\text {crys }, i}$.

Proof. By Proposition 2.10 in fact $\Phi_{\mathscr{P}}$ preserves the ample cone of the closed fiber and so we can choose $\sigma$ such that the map on cohomology is $\theta_{0}$. By Corollary $4.17 \sigma$ lifts uniquely to each infinitesimal neighborhood of $s$ in $B$, and therefore by the Grothendieck existence theorem we get a lifting $\tilde{\sigma}$ over $\widehat{B}$. That the realization of $\tilde{\sigma}$ on cohomology agrees with $\pm \Phi_{\mathscr{P}}^{\text {crys }, i}$ can be verified on the closed fiber where it holds by assumption.

Lemma 6.9. With notation and assumptions as in Proposition 6.8 the map $\Phi_{P}^{A_{\mathrm{num}, \mathbb{Q}}^{*}}$ preserves the ample cones of the generic fibers.

Proof. The statement can be verified after making a field extension of the function field of $B$. The result therefore follows from Proposition 6.8 and Proposition 2.10.

Remark 6.10. In the case when the original $\Phi_{P}$ preserves the ample cones of the geometric generic fibers, no reflections along $(-2)$-curves in the generic fiber are needed. Indeed, by the above argument we get an isomorphism $\sigma_{K}: X_{K} \rightarrow Y_{K}$ such that the induced map on crystalline and étale cohomology agrees with $\Phi_{P} \circ \alpha$ for some sequence $\alpha$ of spherical twists along (-2)-curves in $X_{K}$ (also using Corollary 6.9). Since both $\sigma$ and $\Phi_{P}$ preserve ample 
cones it follows that $\alpha$ also preserves the ample cone of $X_{\bar{K}}$. By [24, 1.10] it follows that $\alpha$ acts trivially on the Néron-Severi group of $X_{\bar{K}}$. We claim that this implies that $\alpha$ also acts trivially on any of the cohomological realizations. We give the proof in the case of étale cohomology $H^{2}\left(X_{\bar{K}}, \mathbb{Q}_{\ell}\right.$ ) (for a prime $\ell$ invertible in $k$ ) leaving slight modifications for the other cohomology theories to the reader. Let $\widetilde{R}_{X}$ denote the subgroup of $G L\left(H^{2}\left(X_{\bar{K}}, \mathbb{Q}_{\ell}\right)\right)$ generated by -1 and the action induced by spherical twists along $(-2)$-curves in $X_{\bar{K}}$, and consider the inclusion of $\mathbb{Q}_{\ell}$-vector spaces with inner products

$$
N S\left(X_{\bar{K}}\right)_{\mathbb{Q}_{\ell}} \hookrightarrow H^{2}\left(X_{\bar{K}}, \mathbb{Q}_{\ell}\right) .
$$

By [11, Lemma 8.12] the action of the spherical twists along $(-2)$-curves in $X_{\bar{K}}$ on $H^{2}\left(X_{\bar{K}}, \mathbb{Q}_{\ell}\right)$ is by reflection across classes in the image of $N S\left(X_{\bar{K}}\right)_{\mathbb{Q}_{\ell}}$. From this (and Gram-Schmidt!) it follows that the the group $\widetilde{R}_{X}$ preserves $N S\left(X_{\bar{K}}\right)_{\mathbb{Q}_{\ell}}$, acts trivially on the quotient of $H^{2}\left(X_{\bar{K}}, \mathbb{Q}_{\ell}\right)$ by $N S\left(X_{\bar{K}}\right)_{\mathbb{Q}_{\ell}}$, and that the restriction map

$$
\widetilde{R}_{X} \rightarrow G L\left(N S\left(X_{\bar{K}}\right)_{\mathbb{Q}_{\ell}}\right)
$$

is injective. In particular, if an element $\alpha \in \widetilde{R}_{X}$ acts trivially on $N S\left(X_{\bar{K}}\right)$ then it also acts trivially on étale cohomology. It follows that $\sigma$ and $\Phi_{P}$ induce the same map on realizations.

\section{Specialization}

7.1. We consider again the setup of Proposition 6.1, but now we don't assume that the closed fiber $X_{s}$ is supersingular. Further we restrict attention to the case when $B$ is a smooth curve, and assume we are given a smooth model $Y / B$ of $Y_{K}$ and a $B$-perfect complex $\mathscr{P} \in D\left(X \times{ }_{B} Y\right)$ such that for all geometric points $\bar{z} \rightarrow B$ the induced complex $\mathscr{P}_{\bar{z}}$ on $X_{\bar{z}} \times Y_{\bar{z}}$ defines a strongly filtered equivalence $D\left(X_{\bar{z}}\right) \rightarrow D\left(Y_{\bar{z}}\right)$.

Let $\mathscr{H}^{i}(X / B)\left(\right.$ resp. $\left.\mathscr{H}^{i}(Y / B)\right)$ denote either $\mathscr{H}_{\text {ét }}^{i}(X / B)\left(\right.$ resp. $\left.\mathscr{H}_{\text {ét }}^{i}(Y / B)\right)$ for some prime $\ell \neq p$ or $\mathscr{H}_{\text {crys }}^{i}(X / B)$ (resp. $\left.\mathscr{H}_{\text {crys }}^{i}(Y / B)\right)$. Assume further given an isomorphism

$$
\sigma_{K}: X_{K} \rightarrow Y_{K}
$$

inducing the map given by restricting

$$
\Phi_{\mathscr{P}}^{i}: \mathscr{H}^{i}(X / B) \rightarrow \mathscr{H}^{i}(Y / B)
$$

to the generic point.

Remark 7.2. If we work with étale cohomology in this setup we could also consider the spectrum of a complete discrete valuation ring instead of $B$, and in particular also a mixed characteristic discrete valuation ring.

Remark 7.3. When the characteristic of $k$ is zero we can also use de Rham cohomology instead of étale cohomology.

Proposition 7.4. The isomorphism $\sigma_{K}$ extends to an isomorphism $\sigma: X \rightarrow Y$.

Proof. We give the argument here for étale cohomology in the case when $B$ is the spectrum of a discrete valuation ring, leaving the minor modifications for the other cases to the reader. 
Let $Z \subset X \times_{B} Y$ be the closure of the graph of $\sigma_{K}$, so $Z$ is an irreducible flat $V$-scheme of dimension 3 and we have a correspondence

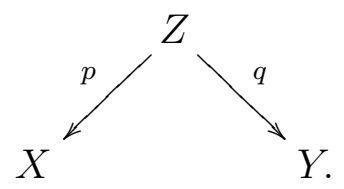

Fix an ample line bundle $L$ on $X$ and consider the line bundle $M:=\operatorname{det}\left(R q_{*} p^{*} L\right)$ on $Y$. The restriction of $M$ to $Y_{K}$ is simply $\sigma_{K *} L$, and in particular the étale cohomology class of $M$ is equal to the class of $\Phi_{\mathscr{P}}(L)$. By our assumption that $\Phi_{\mathscr{P}}$ is strongly filtered in the fibers the line bundle $M$ is ample on $Y$. Note also that by our assumption that $\Phi_{\mathscr{P}}$ is strongly filtered in every fiber we have

$$
\Phi_{\mathscr{P}}\left(L^{\otimes n}\right) \simeq \Phi^{\mathscr{P}}(L)^{\otimes n} .
$$

In particular we can choose $L$ very ample in such a way that $M$ is also very ample. The result then follows from Matsusaka-Mumford [18, Theorem 2].

\section{Proof of Theorem 1.2}

8.1. Let $K$ be an algebraically closed field extension of $k$ and let $X$ and $Y$ be $\mathrm{K} 3$ surfaces over $K$ equipped with a complex $P \in D\left(X \times_{K} Y\right)$ defining a strongly filtered Fourier-Mukai equivalence

$$
\Phi_{P}: D(X) \rightarrow D(Y)
$$

We can then choose a primitive polarization $\lambda$ on $X$ of degree prime to $p$ such that the triple $((X, \lambda), Y, P)$ defines a $K$-point of $\mathscr{S}_{d}$. In this way the proof of Theorem 1.2 is reformulated into showing the following: For any algebraically closed field $K$ and point $((X, \lambda), Y, P) \in$ $\mathscr{S}_{d}(K)$ there exists an isomorphism $\sigma: X \rightarrow Y$ such that the maps on crystalline and étale realizations defined by $\sigma$ and $\Phi_{P}$ agree.

8.2. To prove this it suffices to show that there exists such an isomorphism after replacing $K$ by a field extension. To see this let $I$ denote the scheme of isomorphisms between $X$ and $Y$, which is a locally closed subscheme of the Hilbert scheme of $X \times_{K} Y$. Over $I$ we have a tautological isomorphism $\sigma^{u}: X_{I} \rightarrow Y_{I}$. The condition that the induced action on $\ell$-adic étale cohomology agrees with $\Phi_{P}$ is an open and closed condition on $I$. It follows that there exists a subscheme $I^{\prime} \subset I$ classifying isomorphisms $\sigma$ as in the theorem. This implies that if we can find an isomorphism $\sigma$ over a field extension of $K$ then such an isomorphism also exists over $K$.

8.3. By Proposition 7.4 it suffices to show that the result holds for each generic point of $\mathscr{S}_{d}$. By Theorem 3.9 any such generic point maps to a generic point of $\mathscr{M}_{d}$ which by Theorem 3.2 admits a specialization to a supersingular point $x \in \mathscr{M}_{d}(k)$ given by a family $\left(X_{R}, \lambda_{R}\right) / R$, where $R$ is a complete discrete valuation ring over $k$ with residue field $\Omega$, for some algebraically closed field $\Omega$. By Theorem 5.3 the point $\left(Y, \lambda_{Y}\right) \in \mathscr{M}_{d}(K)$ also has a limit $y \in \mathscr{M}_{d}(\Omega)$ given by a second family $\left(Y_{R}, \lambda_{R}\right) / R$. Let $P^{\prime}$ be the complex on $X \times Y$ giving the composition of $\Phi_{P}$ with suitable twists by $(-2)$-curves such that after replacing $Y_{R}$ by a sequence of flops the map $\Phi_{P^{\prime}}$ induces an isomorphism on crystalline cohomology on the closed fiber preserving plus or minus the ample cone. By the Cohen structure theorem we have $R \simeq \Omega[[t]]$, and $\left((X, \lambda), Y, P^{\prime}\right)$ defines a point of $\mathscr{S}_{d}(\Omega((t)))$. 
Let $B$ denote the completion of the strict henselization of $\mathscr{M}_{\mathbb{Z}[1 / d]} \times \mathscr{M}_{\mathbb{Z}[1 / d]}$ at the point $(x, y)$. So $B$ is a regular complete local ring with residue field $\Omega$. Let $B^{\prime}$ denote the formal completion of the strict henselization of $\overline{\mathscr{S}}_{d, \mathbb{Z}[1 / d]}$ at the $\Omega((t))$-point given by $\left((X, \lambda), Y, P^{\prime}\right)$. So we obtain a commutative diagram

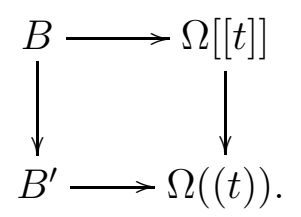

Over $B$ we have a universal families $\mathscr{X}_{B}$ and $\mathscr{Y}_{B}$, and over the base changes to $B^{\prime}$ we have, after trivializing the pullback of the gerbe $\mathscr{S}_{d, \mathbb{Z}[1 / d]} \rightarrow \overline{\mathscr{S}}_{d, \mathbb{Z}[1 / d]}$, a complex $\mathscr{P}_{B^{\prime}}^{\prime}$ on $\mathscr{X}_{B^{\prime}} \times_{B^{\prime}} \mathscr{Y}_{B^{\prime}}$, which reduces to the triple $\left(X, Y, P^{\prime}\right)$ over $\Omega((t))$. The map $B \rightarrow B^{\prime}$ is a filtering direct limit of étale morphisms. We can therefore replace $B^{\prime}$ by a finite type étale $B$-subalgebra over which all the data is defined and we still have the diagram (8.3.1). Let $\bar{B}$ denote the integral closure of $B$ in $B^{\prime}$ so we have a commutative diagram

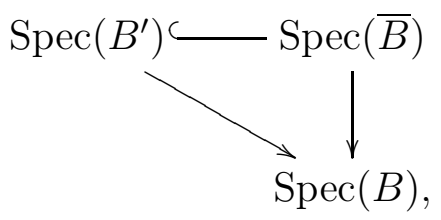

where $\bar{B}$ is flat over $\mathbb{Z}[1 / d]$ and normal. Let $Y \rightarrow \operatorname{Spec}(\bar{B})$ be an alteration with $Y$ regular and flat over $\mathbb{Z}[1 / d]$, and let $Y^{\prime} \subset Y$ be the preimage of $\operatorname{Spec}\left(B^{\prime}\right)$. Lifting the map $B \rightarrow \Omega[[t]]$ to a map $\operatorname{Spec}(\widetilde{R}) \rightarrow Y$ for some finite extension of complete discrete valuation rings $\widetilde{R} / R$ and letting $C$ denote the completion of the local ring of $Y$ at the image of the closed point of $\operatorname{Spec}(\widetilde{R})$ we obtain a commutative diagram

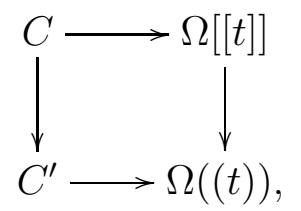

where $C \rightarrow C^{\prime}$ is a localization, we have K3-surfaces $\mathscr{X}_{C}$ and $\mathscr{Y}_{C}$ over $C$ and a perfect complex $\mathscr{P}_{C^{\prime}}^{\prime}$ on $\mathscr{X}_{C^{\prime}} \times_{C^{\prime}} \mathscr{Y}_{C^{\prime}}$ defining a Fourier-Mukai equivalence and the triple $\left(\mathscr{X}_{C^{\prime}}, \mathscr{Y}_{C^{\prime}}, \mathscr{P}_{C^{\prime}}^{\prime}\right)$ reducing to $(X, Y, P)$ over $\Omega((t))$. By [27, 5.2.2] we can extend the complex $\mathscr{P}_{C^{\prime}}^{\prime}$ to a $C$ perfect complex $\mathscr{P}_{C}^{\prime}$ on $\mathscr{X}_{C} \times_{C} \mathscr{Y}_{C}$ (here we use that $C$ is regular). It follows that the base change $\left(X_{\Omega[[t]]}, Y_{\Omega[[t]]}, P_{\Omega[[t]]}^{\prime}\right)$ gives an extension of $(X, Y, P)$ to $\Omega[[t]]$ all of whose reductions satisfy our condition $(\star)$.

This puts us in the setting of Proposition 6.8, and we conclude that there exists an isomorphism $\sigma: X \rightarrow Y$ (over $\Omega((t))$, but as noted above we are allowed to make a field extension of $K$ ) such that the induced map on crystalline and étale cohomology agrees with $\Phi_{P} \circ \alpha$ for some sequence $\alpha$ of spherical twists along (-2)-curves in $X$ (using also Corollary 6.9). By the same argument as in Remark 6.10 it follows that $\sigma$ and $\Phi_{P}$ induce the same map on realizations which concludes the proof of Theorem 1.2 . 
Remark 8.4. One consequence of the proof is that in fact any strongly filtered equivalence automotically takes the ample cone to the ample cone, and not its negative. This is closely related to [12, 4.1].

\section{Characteristic 0}

From our discussion of positive characteristic results one can also deduce the following result in characteristic 0 .

Theorem 9.1. Let $K$ be an algebraically closed field of characteristic 0 , let $X$ and $Y$ be $K 3$ surfaces over $K$, and let $\Phi_{P}: D(X) \rightarrow D(Y)$ be a strongly filtered Fourier-Mukai equivalence defined by an object $P \in D(X \times Y)$. Then there exists an isomorphism $\sigma: X \rightarrow Y$ whose action on $\ell$-adic and de Rham cohomology agrees with the action of $\Phi_{P}$.

Proof. It suffices to show that we can find an isomorphism $\sigma$ which induces the same map on $\ell$-adic cohomology as $\Phi_{P}$ for a single prime $\ell$. For then by compatibility of the comparison isomorphisms with $\Phi_{P}$, discussed in [15, §2], it follows that $\sigma$ and $\Phi_{P}$ also define the same action on the other realizations of the Mukai motive.

Furthermore as in Proposition 8.2 it suffices to prove the existence of $\sigma$ after making a field extension of $K$.

As in Proposition 8.2 let $I^{\prime}$ denote the scheme of isomorphisms $\sigma: X \rightarrow Y$ as in the theorem. Note that since the action of such $\sigma$ on the ample cone is fixed, the scheme $I^{\prime}$ is in fact of finite type.

Since $X, Y$, and $P$ are all locally finitely presented over $K$ we can find a finite type integral $\mathbb{Z}$-algebra $A$, K3 surfaces $X_{A}$ and $Y_{A}$ over $A$, and an $A$-perfect complex $P_{A} \in D\left(X_{A} \times_{A} Y_{A}\right)$ defining a strongly filtered Fourier-Mukai equivalence in every fiber, and such that $(X, Y, P)$ is obtained from $\left(X_{A}, Y_{A}, P_{A}\right)$ by base change along a map $A \rightarrow K$. The scheme $I^{\prime}$ then also extends to a finite type $A$-scheme $I_{A}^{\prime}$ over $A$. Since $I^{\prime}$ is of finite type over $A$ to prove that $I^{\prime}$ is nonempty it suffices to show that $I_{A}^{\prime}$ has nonempty fiber over $\mathbb{F}_{p}$ for infinitely many primes p. This holds by Theorem 1.2.

\section{Bypassing Hodge theORY}

10.1. The appeal to analytic techniques implicit in the results of Section 5 , where characteristic 0 results based on Hodge theory are used to deduce Theorem 5.3. can be bypassed in the following way using results of [19] and [17].

10.2. Let $R$ be a complete discrete valuation ring of equicharacteristic $p>0$ with residue field $k$ and fraction field $K$. Let $X / R$ be a smooth K3 surface with supersingular closed fiber. Let $Y_{K}$ be a K3 surface over $K$ and $P_{K} \in D\left(X_{K} \times Y_{K}\right)$ a perfect complex defining a Fourier-Mukai equivalence $\Phi_{P_{K}}: D\left(X_{\bar{K}}\right) \rightarrow D\left(Y_{\bar{K}}\right)$.

Theorem 10.3. Assume that $X$ admits an ample invertible sheaf $L$ such that $p>L^{2}+4$. Then after replacing $R$ by a finite extension there exists a smooth projective K3 surface $Y / R$ with generic fiber $Y_{K}$. 
Proof. Changing our choice of Fourier-Mukai equivalence $P_{K}$, we may assume that $P_{K}$ is strongly filtered. Setting $M_{K}$ equal to $\operatorname{det}\left(\Phi_{P_{K}}(L)\right)$ or its dual, depending on whether $\Phi_{P_{K}}$ preserves ample cones, we get an ample invertible sheaf on $Y_{K}$ of degree $L^{2}$. By [17, 2.2], building on Maulik's work [19, Discussion preceding 4.9] we get a smooth K3 surface $Y / R$ with $Y$ an algebraic space. Now after replacing $P_{K}$ by the composition with twists along $(-2)$ curves and the model $Y$ by a sequence of flops, we can arrange that the map on crystalline cohomology of the closed fibers induced by $\Phi_{P_{K}}$ preserves ample cones. Let $P \in D\left(X \times{ }_{R} Y\right)$ be an extension of $P_{K}$ and let $M \operatorname{denote} \operatorname{det}\left(\Phi_{P}(L)\right)$. Then $M$ is a line bundle on $Y$ whose reduction is ample on the closed fiber. It follows that $M$ is also ample on $Y$ so $Y$ is a projective scheme.

10.4. We use this to prove Theorem 1.2 in the case of étale realization in the following way. First observe that using the same argument as in Section 8 , but now replacing the appeal to Theorem 5.3 by the above Theorem 10.3 , we get Theorem 1.2 under the additional assumption that $X$ admits an ample invertible sheaf $L$ with $p>L^{2}+4$. By the argument of Section

9 this suffices to get Theorem 1.2 in characteristic 0 , and by the specialization argument of Section 7 we then get also the result in arbitrary characteristic.

\section{REFERENCES}

[1] D. Arinkin and A. Caldararu, When is the self-intersection of a subvariety a fibration, Adv. in Math. 231 (2012), 815-842.

[2] D. Abramovich, A. Corti, and A. Vistoli, Twisted bundles and admissible covers, Commun. Algebra 31 (2003) 3547-3618.

[3] P. Berthelot and A. Ogus, F-isocrystals and de Rham cohomology. I, Inv. Math. 72 (1983), 159-199.

[4] T. Bridgeland, Equivalences of triangulated categories and Fourier-Mukai transforms, Bull. London Math. Soc. 31 (1999), 25-34.

[5] A. Caldararu, The Mukai pairing, I: the Hochschild structure, preprint, math.AG/0308079.

[6] A. Caldararu, The Mukai pairing, II: The Hochschild-Kostant-Rosenberg isomorphism, Adv. in Math. 194 (2005), 34-66.

[7] F. Charles, The Tate conjecture for K3 surfaces over finite fields, Invent. Math. 194 (2013), 119-145.

[8] P. Deligne, Relèvement des surfaces K3 en caractéristique nulle, Prepared for publication by Luc Illusie. Lecture Notes in Math. 868, Algebraic surfaces (Orsay, 1976-78), pp. 58-79, Springer, Berlin-New York, 1981.

[9] J. Dieudonné and A. Grothendieck, Éléments de géométrie algébrique, Inst. Hautes Études Sci. Publ. Math. 4, 8, 11, 17, 20, 24, 28, 32 (1961-1967).

[10] N. Grigg, Deformations of Categories of Coherent Sheaves and Fourier-Mukai transforms, Ph.D. thesis, University of Washington (2013).

[11] D. Huybrechts, Fourier-Mukai transforms in algebraic geometry, Oxford University Press (2006).

[12] D. Huybrechts, E. Macrì and P. Stellari, Derived equivalences of K3 surfaces and orientation, Duke Math. J. 149 (2009), 461-507.

[13] D. Huybrechts and R. Thomas, Deformation-obstruction theory for complexes via Atiyah and KodairaSpencer classes, Math. Ann. 346 (2010), 545-569.

[14] M. Lieblich, Moduli of complexes on a proper morphism, J. Algebraic Geom. 15 (2006), 175-206.

[15] M. Lieblich and M. Olsson, Fourier-Mukai partners of K3 surfaces in positive characteristic, to appear in Ann. Scientifique de l'ENS.

[16] C. Liedtke, Lectures on supersingular K3 surfaces and the crystalline Torelli theorem, preprint (2014).

[17] C. Liedtke and Y. Matsumoto, Good reduction of K3 surfaces, preprint (2015).

[18] T. Matsusaka and D. Mumford, Two fundamental theorems on deformations of polarized varieties, Amer. J. Math. 86 (1964), 668-684.

[19] D. Maulik, Supersingular K3 surfaces for large primes, Duke Math. J. 163 (2014), 2357-2425. 
[20] D. Maulik and B. Poonen, Néron-Severi groups under specialization, Duke Math. J. 161 (2012), 21672206.

[21] E. Macrì and P. Stellari, Infinitesimal derived Torelli theorem for K3 surfaces, Int. Math. Res. Not. (2009).

[22] K. Pera, The Tate conjecture for K3 surfaces in odd characteristic, Inv. Math. 201 (2015), 625-668.

[23] A. Ogus, Singularities of the height strata in the moduli of K3 surfaces, Moduli of abelian varieties (Texel Island, 1999), 325-343, Progr. Math., 195, Birkhäuser, Basel, 2001.

[24] A. Ogus, A Crystalline Torelli Theorem for Supersingular K3 Surfaces, in "Arithmetic and Geometry", Progress in Mathematics 36, Birkhäuser 1983.

[25] A. Ogus, Supersingular K3 crystals, Astérisque 64 (1979), 3-86.

[26] Y. Toda, Deformations and Fourier-Mukai transforms, J. Differential Geom. 81 (2009), 197-224.

[27] R. Thomason, T. Trobaugh, "Higher algebraic K-theory of schemes and of derived categories," in The Grothendieck Festschrift, Vol III, pp. 247-435, Progress in Mathematics, Birkhauser, Boston, 1990. 